\title{
Hyperbolic Numbers in Modeling Genetic Phenomena
}

\author{
Sergey V. Petoukhov ${ }^{1}$ \\ 1 - Mechanical Engineering Research Institute of Russian Academy of Sciences. Russia, \\ 101990, Moscow, M. Kharitonievskiy pereulok, 4, http://eng.imash.ru/, info @imash.ru
}

Comment: Some elements of this article were presented by the author in his keynote speeches at the following conferences: the International Belgrade Bioinformatics Conference 2018 (Belgrade, Serbia, 18-22 June, 2018, http://belbi.bg.ac.rs/); the 2nd International Conference Artificial Intelligence, Medical Engineering, Education (Moscow, Russia, 6-8 October 2018); the 2nd International Conference on Computer Science, Engineering and Education Applications (Kiev, Ukraine, 27-28 January 2019,). Also an author's presentation with elements of this article was done at the 6th International Conference in Code Biology (Friedrichsdorf, Germany, 3-7 June 2019, http://www.codebiology.org/conferences/Friedrichsdorf2019/).

\begin{abstract}
The article is devoted to applications of 2-dimensional hyperbolic numbers and their algebraic $2^{n}$-dimensional extensions in modeling some genetic phenomena. Mathematical properties of hyperbolic numbers and their matrix representations are described in a connection with alphabets of DNA nucleobases, with inherited phyllotaxis phenomena and with the Weber-Fechner law. New methods of algebraic analysis of the harmony of musical works are proposed, taking into account the innate predisposition of people to music. Known data on using hyperbolic rotations, which are particular cases of hyperbolic numbers, in physics and in some biological phenomena, including phyllotaxis laws and structural features of locomotions, are discussed. The hypothesis is put forward that alphabets of eigenvectors of matrix representations of basis units of $2^{n}$-dimensional hyperbolic numbers play a key role in transmitting biological information and that they can be considered as a foundation of coding information at different levels of biological organization. The proposed algebraic approach is connected with the theme of a grammar of biology. Applications of hyperbolic numbers reveal hidden interrelations between structures of different biological and physical phenomena. They lead to new approaches in mathematical modeling genetic phenomena and innate biological structures.
\end{abstract}

Keywords: hyperbolic numbers, matrix, eigenvectors, genetics, Fibonacci numbers, phyllotaxis, music harmony, DNA.

\section{Contents}

1. Introduction

2. Matrix representations of DNA alphabets and hyperbolic numbers

3. Hyperbolic and Fibonacci numbers in phyllotaxis modeling

4. Fibonacci sequences of $2^{n}$-dimensional hyperbolic numbers

5. Hyperbolic numbers and the Weber-Fechner law

6. The alphabets of orthogonal vector bases associated with basis units of $2^{n}$-dimensional hyperbolic numbers

7. Quint ratios in DNA parameters and musical harmony

8. Applications of algebras of $2^{n}$-dimensional hyperbolic numbers in musicology

9. Advantages of matrix representations of hyperbolic numbers 
2

10. $2^{n}$-dimensional hyperbolic numbers and phenomenological rules of probabilities in genetics

11. Fractal-like multi-dimensional configurational spaces of hyperbolic types

12. Pythagoras and the importance of the concept of number

13. Some concluding remarks

Appendix I. Dyadic groups of binary numbers and matrices of dyadic shifts

Acknowledgments

References

\section{Introduction}

The main task of the mathematical natural sciences is the creation of mathematical models of natural systems. Development of models and formalized theories depends highly on those mathematical notions and instruments, on which they are based. Modern science knows that different natural systems could possess their own individual geometries and their own individual arithmetic [Kline, 1982]. Various kinds of multi-dimensional numbers complex numbers, hyperbolic numbers, dual numbers, quaternions and other hypercomplex numbers - are used in different branches of modern science. They have played the role of the magic tool for development of theories and calculations in problems of heat, light, sounds, fluctuations, elasticity, gravitation, magnetism, electricity, current of liquids, quantummechanical phenomena, special theory of relativity, nuclear physics, etc. For example, in physics thousands of works - only in XX century - were devoted to quaternions of Hamilton (their bibliography is in [Gsponer, Hurni, 2008].

The idea about special mathematical peculiarities of living matter exists long ago. For example V.I. Vernadsky put forward the hypothesis on a non-Euclidean geometry of living nature [Vernadsky, 1965]. It seems an important task to investigate what systems of multi-dimensional numbers are connected or can be connected with ensembles of parameters of the genetic code and inherited biological peculiarities. Some results of such investigation are presented in this article. They are connected with hyperbolic numbers and their algebraic extensions, matrix forms of which give a new class of mathematical models in biology. Author's results described in this article are related in particularly to works by O. Bodnar who noted that ontogenetic transformations of phyllotaxis lattices in plants can be formaly modelled by hyperbolic rotations, which are particular cases of hyperbolic numbers and are well known in the special theory of relativity (Lorentz transformations) [Bodnar, 1992, 1994]. On this basis he stated that geometry of living bodies has structural relations with the Minkovsky geometry. Another evidence in favor of structural relaltions of inherited biological phenomena with hyperbolic rotations was shown in the work [Smolyaninov, 2000], which analyzed problems of locomotion control and put forward ideas of the "locomotor theory of relativity".

It is obvoius that all physiological systems must be argued with a genetic coding system in order to be genetically encoded for their survival and inheritance into next generations. For this reason, the structural organization of physiological systems can bear the imprint of the structural features of molecular genetic systems. Our study aims to identify such relationships of inherited physiological structures with the molecular genetic system. Taking into account known data about ratios of musical harmony in parametric organization of DNA molecules, new algebraic approaches are proposed for analyzing hidden harmony of musical pieces. 


\section{Matrix representations of DNA alphabets and hyperbolic numbers}

In DNA molecules DNA genetic information is written in sequences of 4 kinds of nucleobases: adenine A, cytosine $\mathrm{C}$, guanine $\mathrm{G}$ and thymine $\mathrm{T}$. They form a DNA alphabet of 4 monoplets. In addition, DNA alphabets of 16 doublets and 64 triplets also exist. It is known [Fimmel, Danielli, Strüngmann, 2013; Petoukhov, 2008; Petoukhov, He, 2010; Stambuk, 1999] that these four nucleobases $A, C, G$ and $T$ are interrelated due to their symmetrical peculiarities into the united molecular ensemble with its three pairs of binary-oppositional traits or indicators (Fig. 2.1):

1) Two letters are purines ( $\mathrm{A}$ and $\mathrm{G})$, and the other two are pyrimidines $(\mathrm{C}$ and $\mathrm{T})$. From the standpoint of these binary-oppositional traits one can denote $\mathrm{C}=\mathrm{T}=0, \mathrm{~A}=\mathrm{G}=1$;

2) Two letters are amino-molecules $(\mathrm{A}$ and $\mathrm{C}$ ) and the other two are keto-molecules ( $\mathrm{G}$ and $\mathrm{T})$. From the standpoint of these traits one can designate $\mathrm{A}=\mathrm{C}=0, \mathrm{G}=\mathrm{T}=1$;

3) The pairs of complementary letters, A-T and C-G, are linked by 2 and 3 hydrogen bonds, respectively. From the standpoint of these binary traits, one can denote $C=G=0$, $\mathrm{A}=\mathrm{T}=1$.

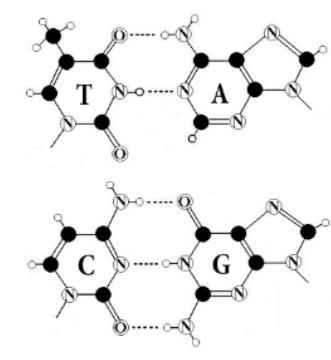

\begin{tabular}{|c|l|c|c|c|c|}
\hline № & \multicolumn{1}{|c|}{ Binary Symbols } & $\mathbf{C}$ & $\mathbf{A}$ & $\mathbf{G}$ & $\mathbf{T} / \mathbf{U}$ \\
\hline 1 & $\begin{array}{l}\mathbf{0}_{1}-\text { pyrimidines } \\
\mathbf{1}_{1}-\text { purines }\end{array}$ & $\mathbf{0}_{1}$ & $\mathbf{1}_{1}$ & $\mathbf{1}_{1}$ & $\mathbf{0}_{1}$ \\
\hline 2 & $\begin{array}{l}\mathbf{0}_{2}-\text { amino } \\
\mathbf{1}_{2}-\text { keto }\end{array}$ & $\mathbf{0}_{2}$ & $\mathbf{0}_{2}$ & $\mathbf{1}_{2}$ & $\mathbf{1}_{2}$ \\
\hline 3 & $\begin{array}{l}\mathbf{0}_{3}-\text { three hydrogen bonds; } \\
\mathbf{1}_{3}-\text { two hydrogen bonds }\end{array}$ & $\mathbf{0}_{3}$ & $\mathbf{1}_{3}$ & $\mathbf{0}_{3}$ & $\mathbf{1}_{3}$ \\
\hline
\end{tabular}

Fig. 2.1. Left: the four nitrogenous bases of DNA: adenine A, guanine G, cytosine C, and thymine T. Right: three binary sub-alphabets of the genetic alphabet on the basis of three pairs of binary-oppositional traits or indicators.

Taking into account the phenomenological fact that each of DNA-letters C, A, T and G is uniquely defined by any two kinds of mentioned binary-oppositional indicators (Fig. 2.1), these genetic letters can be represented by means of corresponding pairs of binary symbols, for example, from the standpoint of two first binary-oppositional indicators. It is convenient for us - for the further description - use at the first position of each of letters its binary symbol from the second pair of binary-oppositional indicators (the indicator "amino or keto": $\mathrm{C}=\mathrm{A}=0, \mathrm{~T}=\mathrm{G}=1$ ) and at the second positions of each of letters its binary symbol from the first pair of binary-oppositional indicators (the indicator "pyrimidine or purine": $C=T=0, A=G=1$ ). In this case the letter $C$ is represented by the binary symbol $0_{2} 0_{1}$ (that is as 2-bit binary number), $\mathrm{A}-$ by the symbol $0_{2} 1_{1}, \mathrm{~T}-$ by the symbol $1_{2} 0_{1}, \mathrm{G}-$ by the symbol $1_{2} 1_{1}$. Using these representations of separate letters, each of 16 doublets is represented as the concatenation of the binary symbols of its letters (that is as 4-bit binary number): for example, the 
4

doublet $\mathrm{CC}$ is represented as 4-bit binary number $0_{2} \mathrm{O}_{1} \mathrm{O}_{2} \mathrm{O}_{1}$, the doublet $\mathrm{CA}$ - as 4-bit binary number $0_{2} 0_{1} 0_{2} 1_{1}$, etc. By analogy, each of 64 triplets is represented as the concatenation of the binary symbols of its letters (that is as 6-bit binary number): for example, the triplet CCC is represented as 6-bit binary number $0_{2} 0_{1} 0_{2} 0_{1} O_{2} O_{1}$, the triplet CCA - as 6-bit binary number $\mathrm{O}_{2} \mathrm{O}_{1} \mathrm{O}_{2} \mathrm{O}_{1} \mathrm{O}_{2} 1_{1}$, etc. In general, each of n-plets is represented as the concatenation of the binary symbols of its letters (below we will not show these indexes 2 and 1 of separate letters in binary representations of n-plets but will remember that each of positions corresponds to its own kind of indicators from the first or from the second set of indicators in Fig. 2.1).

It is convenient to represent DNA-alphabets of 4 nucleotides, 16 doublets, 64 triplets, ... $4^{\mathrm{n}} \mathrm{n}$-plets in a form of appropriate square tables (Fig. 2.2), which rows and columns are numerated by binary symbols in line with the following principle. Entries of each column are numerated by binary symbols in line with the first set of binary-oppositional indicators in Fig. 2.1 (for example, the triplet CAG and all other triplets in the same column are the combination "pyrimidine-purin-purin" and so this column is correspondingly numerated 011). By contrast, entries of each of rows are numerated by binary numbers in line with the second set of indicators (for example, the same triplet CAG and all other triplets in the same row are the combination "amino-amino-keto" and so this row is correspondingly numerated 001). In such tables (Fig. 2.2), each of 4 letters, 16 doublets, 64 triplets, ... takes automatically its own individual place and all components of the alphabets are arranged in a strict order.

It is essential that these 3 separate genetic tables form the joint tensor family of matrices since they are interrelated by the known operation of the tensor (or Kronecker) product of matrices [Bellman, 1960]. So they are not simple tables but matrices. By definition, under tensor multiplication of two matrices, each of entries of the first matrix is multiplied with the whole second matrix. The second tensor power of the $(2 * 2)$-matrix [C, A; T, G] of 4 DNAletters gives automatically the (4*4)-matrix of 16 doublets; the third tensor power of the same $(2 * 2)$-matrix of 4 DNA-letters gives the $(8 * 8)$-matrix of 64 triplets with the same strict arrangement of entries as in Fig. 2.2. In this tensor construction of the tensor family of genetic matrices, data about binary-oppositional traits of genetic letters $\mathrm{C}, \mathrm{A}, \mathrm{T}$ and $\mathrm{G}$ are not used at all. So, the structural organization of the system of DNA-alphabets is connected with the algebraic operation of the tensor product. It is important since the operation of the tensor product is well known in mathematics, physics and informatics, where it gives a way of putting vector spaces together to form larger vector spaces. The following quotation speaks about the crucial meaning of the tensor product: «This construction is crucial to understanding the quantum mechanics of multiparticle systems» [Nielsen, Chuang, 2010, p. 71].

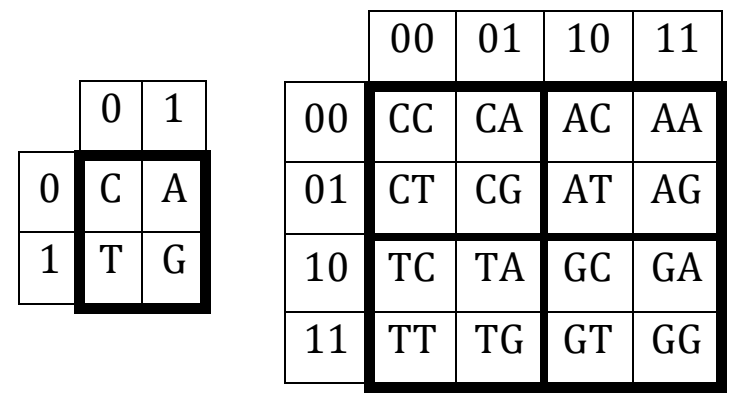




\begin{tabular}{|c|l|l|l|l|l|l|l|l|}
\cline { 2 - 8 } \multicolumn{1}{c|}{} & 000 & 001 & 010 & 011 & 100 & 101 & 110 & 111 \\
\hline 000 & CCC & CCA & CAC & CAA & ACC & ACA & AAC & AAA \\
\hline 001 & CCT & CCG & CAT & CAG & ACT & ACG & AAT & AAG \\
\hline 010 & CTC & CTA & CGC & CGA & ATC & ATA & AGC & AGA \\
\hline 011 & CTT & CTG & CGT & CGG & ATT & ATG & AGT & AGG \\
\hline 100 & TCC & TCA & TAC & TAA & GCC & GCA & GAC & GAA \\
\hline 101 & TCT & TCG & TAT & TAG & GCT & GCG & GAT & GAG \\
\hline 110 & TTC & TTA & TGC & TGA & GTC & GTA & GGC & GGA \\
\hline 111 & TTT & TTG & TGT & TGG & GTT & GTG & GGT & GGG \\
\hline
\end{tabular}

Fig. 2.2. The square tables of DNA-alphabets of 4 nucleotides, 16 doublets and 64 triplets with a strict arrangement of all components. Each of tables is constructed in line with the principle of binary numeration of its column and rows on the basis of binary-oppositional traits of the nitrogenous bases (see explanations in the text).

In the DNA double helix, complementary nucleobases $\mathrm{C}$ and $\mathrm{G}$ are connected by 3 hydrogen bonds and complementary nucleobases $\mathrm{A}$ and $\mathrm{T}$ are connected by 2 hydrogen bonds. One can denote their typical connections with hydrogen bonds by expressions $\mathrm{C}=\mathrm{G}=3$ and $\mathrm{A}=\mathrm{T}=2$. Replacing in the (2*2)-matrix [C, A; T, G] (Fig. 2.2) symbols C, A, T and G by their numbers of hydrogen bonds 3 and 2, a numeric matrix [3, 2; 2, 3] appears (Fig. 2.3). The second and the third tensor powers of this matrix $[3,2 ; 2,3]^{(\mathrm{n})}$, where $n=2,3$, generate numeric (4*4)- and (8*8)-matrices in Fig. 2.3, which automatically represent symbolic matrices of 16 doublets and 64 triplets in Fig. 2.2 from the standpoint of the product of their numbers of hydrogen bonds. For example the doublet CA is replaced by number $3 * 2=6$ and the triplet AGT is replaced by number $2 * 3 * 2=12$. These genetic matrices are closely connected by their structures with so called matrices of dyadic shifts, which are known in digital information technology of noise immune coding and which are described below in the Appendix I.

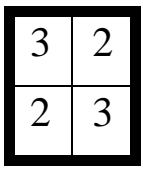

\begin{tabular}{|l|l|l|l|}
\hline 9 & 6 & 6 & 4 \\
\hline 6 & 9 & 4 & 6 \\
\hline 6 & 4 & 9 & 6 \\
\hline 4 & 6 & 6 & 9 \\
\hline
\end{tabular}




\begin{tabular}{|c|c|c|c|c|c|c|c|}
\hline 27 & 18 & 18 & 12 & 18 & 12 & 12 & 8 \\
\hline 18 & 27 & 12 & 18 & 12 & 18 & 8 & 12 \\
\hline 18 & 12 & 27 & 18 & 12 & 8 & 18 & 12 \\
\hline 12 & 18 & 18 & 27 & 8 & 12 & 12 & 18 \\
\hline 18 & 12 & 12 & 8 & 27 & 18 & 18 & 12 \\
\hline 12 & 18 & 8 & 12 & 18 & 27 & 12 & 18 \\
\hline 12 & 8 & 18 & 12 & 18 & 12 & 27 & 18 \\
\hline 8 & 12 & 12 & 18 & 12 & 18 & 18 & 27 \\
\hline
\end{tabular}

Fig. 2.3. Numeric representations of the tensor family of symbolic matrices (Fig. 2.2) of 4 monoplets, 16 doublets and 64 triplets from the standpoint of their numeric characteristics of hydrogen bonds $\mathrm{C}=\mathrm{G}=3$ and $\mathrm{A}=\mathrm{T}=2$.

Fig. 2.4 shows that the matrix $[3,2 ; 2,3]$ is decomposed into sum of two sparse matrices, one of which is the identity matrix $\left(\mathrm{j}_{0}=[1,0 ; 0,1]\right)$ and the second matrix $\left.\mathrm{j}_{1}=[0,1 ; 1,1]\right)$ represents imaginary unit of hyperbolic numbers since $\mathrm{j}_{1}{ }^{2}=\mathrm{j}_{0}$. The set of these matrices $\mathrm{j}_{0}$ and $\mathrm{j}_{1}$ is closed relative to multiplication and defines the multiplication table of algebra of hyperbolic numbers (Fig. 2.4, right).

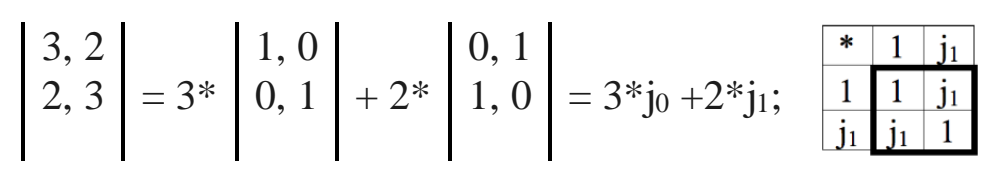

Fig. 2.4. The decomposition of the matrix $[3,2 ; 2,3]$ into two sparse matrices, where matrices $\mathrm{j}_{0}$ and $\mathrm{j}_{1}$ are matrix representations of real and imaginary units of algebra of hyperbolic numbers with the shown multiplication table of these units.

Here we should remind that two-dimensional hyperbolic numbers are written in linear notation as $m_{l}=a^{*} \mathbf{1}+b^{*} \mathbf{j}$ (where $\mathbf{1}$ is the real unit; $\mathbf{j}$ is the imaginary unit with the property $\mathbf{j} \neq \pm \mathbf{1}$ but $\mathbf{j}^{2}=\mathbf{1} ; a, b$ are real coefficients). These numbers are used in physics and mathematics and they have also synonimical names: "split-complex numbers", "perplex numbers" and "double numbers". The collection of all hyperbolic numbers forms algebra over the field of real numbers [Harkin, Harkin, 2004; Kantor, Solodovnikov, 1989]. The algebra is not a division algebra or field since it contains zero divisors. Addition and multiplication of hyperbolic numbers are defined by (2.1):

$$
(x+j y)+(u+j v)=(x+u)+j(y+v) ; \quad(x+j y)(u+j v)=(x u+y v)+j(x v+y u)
$$


This multiplication is commutative, associative and distributes over addition.

A hyperbolic number has its matrix form of representation: $[a, b ; b, a]=a^{*}[1,0 ; 0,1]$ $+b^{*}[0,1 ; 1,0]$ where $[1,0 ; 0,1]$ is the identity matrix representing real basis unit; $[0,1 ; 1,0]$ represents imaginary basis unit. Fig. 2.4 shows the matrix representation of hyperbolic numbers $a^{*} \mathbf{1}+b^{*} \mathbf{j}$ for the case $a=3$ and $b=2$. The symmetric matrices $[1,0 ; 0,1]$ and $[0,1 ; 1,0]$ representing these real and imaginary unites are orthogonal matrices.

If $a^{2}-b^{2}=1$, then the matrix [a, $\left.\mathrm{b} ; \mathrm{b}, \mathrm{a}\right]$ defines hyperbolic rotations known in the special theory of relativity as Lorentz transformations. Hyperbolic rotations are usually expressed by a symmetric matrix (2.2) through hyperbolic cosine «cosh» and hyperbolic sine «sinh» since $\cosh ^{2} x-\sinh ^{2} x=1$ [Collins Concise Dictionary, 1999; Shervatov, 1954; Stakhov, 2009]:

$$
\left|\begin{array}{cc}
\cosh ^{2} a, & \sinh ^{2} b \\
\sinh ^{2} b, & \cosh ^{2} a
\end{array}\right|
$$

Symmetric matrices that represent hyperbolic numbers have real eigenvalues and orthogonal eigenvectors (which distinguishes them from non-symmetic matrix representations of complex numbers). Such symmetric matrices form the basis of the theory of resonances of oscillatory systems with many degrees of freedom, and are also metric tensors from the point of view of Riemannian geometry.

The second tensor power of the bisymmetric matrix $[a, b ; b, a]$, which represents hyperbolic numbers, is decomposed into 4 sparse matrices $\mathrm{e}_{0}, \mathrm{e}_{1}, \mathrm{e}_{2}$ and $\mathrm{e}_{3}$ with real coefficients $a a, a b b a$ and $b b$ (Fig. 2.5). The used decomposition is based on the known principle of dyadic shifts described below in the Appendix I.

The set of matrices $\mathrm{e}_{0}, \mathrm{e}_{1}, \mathrm{e}_{2}$ and $\mathrm{e}_{3}$ is closed relative to multiplication and satisfies to the multiplication table in Fig. 2.5. The set of these $(4 \times 4)$-matrices corresponds to algebra of 4-dimensional numbers $a a^{*} \mathrm{e}_{0}+a b^{*} \mathrm{e}_{1}+b a^{*} \mathrm{e}_{2}+b b^{*} \mathrm{e}_{3}$, where the matrix $\mathrm{e}_{0}$ represents the real unit 1 and matrices $e_{1}, e_{2}$ and $e_{3}$ represent imaginary units. These 4-dimensional numbers are algebraic extensions of 2-dimensional hyperbolic numbers and for simplicity they can be termed "4-dimensional hyperbolic numbers" (in our previous publications we termed them "hyperbolic matrions" [Petoukhov, 2008; Petoukhov, He, 2010]). Each of matrices $\mathrm{e}_{0}, \mathrm{e}_{1}, \mathrm{e}_{2}$ and $e_{3}$ is an orthogonal matrix with its determinant +1 .

By comparing Fig. 2.3 and Fig. 2.5, one can see that the numeric (4*4)-matrix of hydrogen bonds in Fig. 2.3 represents 4-dimensional hyperbolic number $9 \mathrm{e}_{0}+6 \mathrm{e}_{1}+6 \mathrm{e}_{2}+4 \mathrm{e}_{3}$ where $\mathrm{e}_{0}$ is the identity matrix representing real unit 1 . By analogy, the numeric $(8 * 8)$-matrix in Fig. 2.3 represents 8 -dimensional hyperbolic number $27 \mathrm{j}_{0}+18 \mathrm{j}_{1}+18 \mathrm{j}_{2}+12 \mathrm{j}_{3}+18 \mathrm{j}_{4}+12 \mathrm{j}_{5}+12 \mathrm{j}_{6}+8 \mathrm{j}_{7}$ where $\mathrm{j}_{\mathrm{k}}$ are basis units of 8-dimensional hyperbolic niumbers. 


$$
\begin{aligned}
& \left|\begin{array}{l}
a, b \\
b, a
\end{array}\right|=\left|\begin{array}{l}
a a, a b, b a, b b \\
a b, a b, b b, b a \\
b a, b b, a b, a b \\
b b, b a, a b, a a
\end{array}\right|=a a\left|\begin{array}{llll}
1 & 0 & 0 & 0 \\
0 & 1 & 0 & 0 \\
0 & 0 & 1 & 0 \\
0 & 0 & 0 & 1
\end{array}\right|+a b\left|\begin{array}{llll}
0 & 1 & 0 & 0 \\
1 & 0 & 0 & 0 \\
0 & 0 & 0 & 1 \\
0 & 0 & 1 & 0
\end{array}\right|+b a\left|\begin{array}{llll}
0 & 0 & 1 & 0 \\
0 & 0 & 0 & 1 \\
1 & 0 & 0 & 0 \\
0 & 1 & 0 & 0
\end{array}\right|+
\end{aligned}
$$

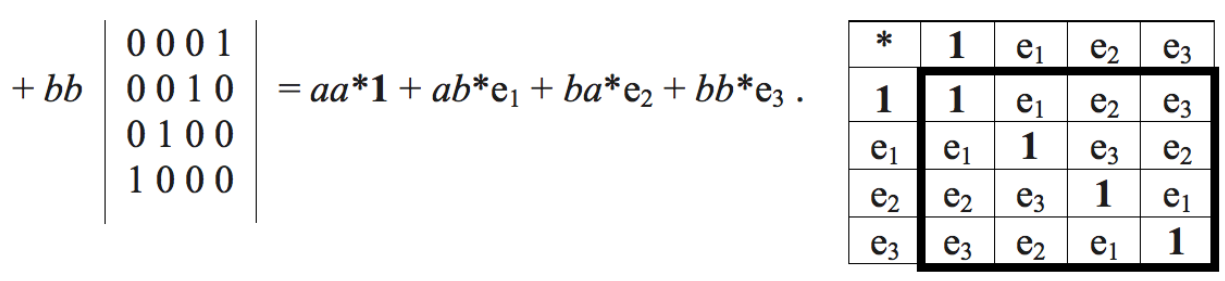

Fig. 2.5. The decomposition of the matrix $[a, b ; b, a]{ }^{(2)}$, representing 4-dimensional hyperbolic numbers, into 4 sparse matrices, the set of which is closed relative to multiplication. The multiplication table for this set is shown at the right. The symbol $\mathbf{1}$ denotes the identity matrix $\mathrm{e}_{0}$.

In a general case, $2^{n}$-dimensional hyperbolic numbers are hypercomplex numbers and they possess, by definition, the following features. They contain $2^{n}$ basis units $\mathrm{e}_{k}$ (one real unit and $2^{n}$-1 imaginary units), which are interrelated by a symmetric table of their mutual multiplication where all $\mathrm{e}_{k}^{2}=+1\left(k=0,1,2, \ldots, 2^{n}-1\right)$.

By analogy with Figs. 2.4 and 2.5, the higher tensor powers $n=3,4,5, \ldots$ of the bisymmetric matrix $[a, b ; b, a]$ produce bisymmetric matrices $[a, b ; b, a]{ }^{(n)}$, which can be also decomposed into $2^{n}$ sparse matrices, the set of which is closed relative to multiplication and which define appropriate multiplication tables of algebras of $2^{n}$-dimensional hypercomplex numbers $m_{n}$ (which were termed "hyperbolic matrions" of the order $n$ in our previous publications [Petoukhov 2008; Petoukhov, He, 2010]). These decompositions use a structural similarity of the matrices $[a, b ; b, a]{ }^{(n)}$ with matrices of dyadic shifts described below in the Appendix I.

It is useful to rewrite the multiplication table in Fig. 2.5 into a form where all decimal indexes of basis units $\mathrm{e}_{0}, \mathrm{e}_{1}, \mathrm{e}_{2}$ and $\mathrm{e}_{3}$ are shown in their binary notations: $\mathrm{e}_{00}, \mathrm{e}_{01}, \mathrm{e}_{10}$ and $\mathrm{e}_{11}$ (Fig. 2.6).

\begin{tabular}{|c|c|c|c|c|}
\hline$*$ & $\mathrm{e}_{00}$ & $\mathrm{e}_{01}$ & $\mathrm{e}_{10}$ & $\mathrm{e}_{11}$ \\
\hline $\mathrm{e}_{00}$ & $\mathrm{e}_{00}$ & $\mathrm{e}_{01}$ & $\mathrm{e}_{10}$ & $\mathrm{e}_{11}$ \\
\hline $\mathrm{e}_{01}$ & $\mathrm{e}_{01}$ & $\mathrm{e}_{00}$ & $\mathrm{e}_{11}$ & $\mathrm{e}_{10}$ \\
\hline $\mathrm{e}_{10}$ & $\mathrm{e}_{10}$ & $\mathrm{e}_{11}$ & $\mathrm{e}_{00}$ & $\mathrm{e}_{01}$ \\
\hline $\mathrm{e}_{11}$ & $\mathrm{e}_{11}$ & $\mathrm{e}_{10}$ & $\mathrm{e}_{01}$ & $\mathrm{e}_{00}$ \\
\hline
\end{tabular}

Fig. 2.6. The multiplication table in algebra of 4-dimensional hyperbolic numbers where indexes of basis units are shown in their binary notations $\mathrm{e}_{00}, \mathrm{e}_{01}, \mathrm{e}_{10}$ and $\mathrm{e}_{11}$ in contrast to their decimal notations $\mathrm{e}_{0}, \mathrm{e}_{1}, \mathrm{e}_{2}$ and $\mathrm{e}_{3}$ in Fig. 2.5.

One can see from Fig. 2.6 that in all cases a result of the product of two basis units $\left(\mathrm{e}_{p} * \mathrm{e}_{k}=\mathrm{e}_{s}\right)$ is equal to that basis unit $\mathrm{e}_{s}$ whose binary index $s$ is equal to a result of modulo-2 addition for binary indexes $p$ and $k$ of the factors $\mathrm{e}_{p}$ and $\mathrm{e}_{k}$ (under the operation of modulo-2 addition the following rules are true: $0+0=0,1+1=0,0+1=1,1+0=1)$. In other words the following equation (2.3) for bimary indexes is true: 


$$
\mathrm{e}_{p} * \mathrm{e}_{k}=\mathrm{e}_{p+k}
$$

For example, a result of the product $\mathrm{e}_{2} *_{3} \mathrm{e}_{3}$ is equal to $\mathrm{e}_{1}$ since decimal indexes 2 and 3 are expressed by binary numbers 10 and 11 whose modulo- 2 addition gives the binary number 01 refered to decimal number 1 . This method of binary operations with indexes to calculate a result of the product of any two basis units is true not only for 4-dimensional hyperbolic numbers but also for other $2^{n}$-dimensional hyperbolic numbers. The equation (2.3) is especially useful in cases of high values $n$ when it is difficult to address to multiplication tables having $2^{n *} 2^{n}$ sizes each time when you need to know a result $\mathrm{e}_{s}$ of the product of basis units $\mathrm{e}_{p} * \mathrm{e}_{k}=\mathrm{e}_{s}$.

For this you should represent indexes $p$ and $k$ in their binary notation (inside a complete set of $n$-bit binary numbers) and calculate their binary sum $p+k$ on the basis of the known operation of modulo- 2 addition where the following rules are true: $0+0=0,1+1=0,0+1=1,1+0=1$. The result of such modulo- 2 addition is a searched index $s$ in its binary notation. For example, if you multiplicate two $2^{3}$-dimensional hyperbolic numbers each other, the complete set of 3-bit binary numbers is the following: 000, 001, 010, 011, 100, 101, 110, 111 (they correspond decimal numbers $0,1,2,3,4,5,6,7)$. To calculate a result of multuplication of basis units $\mathrm{e}_{3}{ }^{*} \mathrm{e}_{5}$, you take decimal indexes 3 and 5 in their binary notation 011 and 101 . Their modulo- 2 addition gives binary number 110 , which corresponds decimal number 6 . In such way we get the search result: $\mathrm{e}_{3} * \mathrm{e}_{5}=\mathrm{e}_{6}$.

\section{$3 \quad$ Hyperbolic and Fibonacci numbers in phyllotaxis modeling}

Fibonacci numbers $\mathrm{F}_{n}$ form an additive sequence such that each number is the sum of the two preceding ones: $\mathrm{F}_{n}=\mathrm{F}_{n-1}+\mathrm{F}_{n-2}$ (Table 3.1).

Table 3.1. The Fibonacci sequence.

\begin{tabular}{|l|l|l|l|l|l|l|l|l|l|l|l|}
\hline$n$ & 1 & 2 & 3 & 4 & 5 & 6 & 7 & 8 & 9 & 10 & $\ldots$ \\
\hline $\mathrm{F}_{n}$ & 1 & 1 & 2 & 3 & 5 & 8 & 13 & 21 & 34 & 55 & $\ldots$ \\
\hline
\end{tabular}

Fibonacci numbers are strongly related to the golden ratio $\varphi=\left(1+5^{0.5}\right) / 2$. Binet's formula (3.1) expresses the $n$th Fibonacci number in terms of $n$ and the golden ratio, and implies that the ratio of two consecutive Fibonacci numbers tends to the golden ratio as $n$ increases:

$$
\mathrm{F}_{n}=\left(\varphi^{n}-\left(-\varphi^{-1}\right)\right) / 5^{0.5}
$$

In biology, it has long been known that, for example, in many plant objects the spiral arrangement of their bioorganisms form ordered patterns (shoots of plants and trees, seeds in the heads of sunflowers, scales of coniferous cones and pineapples, etc.). These patterns are determined by overlapping left and right oriented spiral lines - parastichies. To characterize phyllotaxis of such botanical objects, usually indicate two parameters: number of left spirals and number of right spirals, which are observed on the surface of phyllotaxis objects. 
10

Phyllotaxis of structures with such patterns is described by ratios of neighboring Fibonacci numbers:

$$
\begin{gathered}
\mathrm{F}_{n+1} / \mathrm{F}_{n}: 2 / 1,3 / 2,5 / 3,8 / 5,13 / 8,21 / 13,34 / 21, \ldots \\
\left(\mathrm{F}_{n+1} / \mathrm{F}_{n}\right) \rightarrow\left(\mathrm{F}_{n+2} / \mathrm{F}_{n+1}\right): 2 / 1 \rightarrow 3 / 2 \rightarrow 5 / 3 \rightarrow 8 / 5 \rightarrow 13 / 8 \rightarrow 21 / 13 \rightarrow \ldots
\end{gathered}
$$

The sequence (3.2) is termed the "parastichic sequence" [Jean ,2006; Petoukhov, 1981]. It seems natural to use 2-dimensional hyperbolic numbers for modeling these 2-parametric patterns in phyllotaxis objects and their ontogenetic transformations. In this approach, proposed by the author, the sequence (3.2) of phyllotaxis ratios is transformed into additive sequences $(3.4,3.5)$ reflecting linear notation of appropriate hyperbolic numbers and their matrix representations (we call sequences $(3.4,3.5)$ as parastichic sequences of hyperbolic numbers):

$$
\begin{gathered}
\mathrm{F}_{n+1}+\mathrm{j} \mathrm{F}_{n}: 2+\mathrm{j}, 3+\mathrm{j} 2,5+3 \mathrm{j}, 8+5 \mathrm{j}, 13+8 \mathrm{j}, 21+13 \mathrm{j}, 34+21 \mathrm{j}, \ldots \\
\left|\begin{array}{l}
\mathrm{F}_{n+1}, \mathrm{~F}_{n} \\
\mathrm{~F}_{n}, \mathrm{~F}_{n+1}
\end{array}\right|:\left|\begin{array}{l}
2,1 \\
1,2
\end{array}\right|,\left|\begin{array}{l}
3,2 \\
2,3
\end{array}\right|,\left|\begin{array}{l}
5,3 \\
3,5
\end{array}\right|,\left|\begin{array}{c}
8,5 \\
5,8
\end{array}\right|,\left|\begin{array}{c}
13,8 \\
8,13
\end{array}\right| \ldots
\end{gathered}
$$

In this approach, to define a hyperbolic number $\mathrm{u}+\mathrm{jv}$, which transforms a hyperbolic number $\mathrm{F}_{n+1}+\mathrm{j} \mathrm{F}_{n}$ into its neighboring hyperbolic number $\mathrm{F}_{n+2}+\mathrm{j} \mathrm{F}_{n+1}$ from the sequence (3.4), the following simple equation (3.6) should be solved:

$$
\left(\mathrm{F}_{n+1}+\mathrm{j} \mathrm{F}_{n}\right)(\mathrm{u}+\mathrm{jv})=\left(\mathrm{F}_{n+2}+\mathrm{j} \mathrm{F}_{n+1}\right)
$$

The solution to this equation (3.6) gives the following expressions (3.7) for components of the desired hyperbolic number $\mathrm{u}+\mathrm{jv}$ :

$$
\mathrm{u}=\mathrm{F}_{n+1} / \mathrm{F}_{n}+(-1)^{n+1} * \mathrm{~F}_{n-1} /\left(\mathrm{F}_{n} *\left(\mathrm{~F}_{n}^{2}-\mathrm{F}_{n-1}^{2}\right)\right), \quad \mathrm{v}=(-1)^{n} /\left(\mathrm{F}_{n}^{2}-\mathrm{F}_{n-1}{ }^{2}\right)
$$

In the case of such components (3.7), $\mathrm{u}^{2}-\mathrm{v}^{2} \neq 1$ and the appropriate matrix $[\mathrm{u}, \mathrm{v} ; \mathrm{v}, \mathrm{u}]$ does not present a hyperbolic rotation in the sense of expression (2.2). But this matrix can be rewriting into the form (10) where the matrix of a hyperbolic rotation (in the sense of expression (2.2)) is multiplied by a coefficient $\left(u^{2}-v^{2}\right)^{0.5}$ :

$$
[u, v ; v, u]=\left(u^{2}-v^{2}\right)^{0.5}\left[u\left(u^{2}-v^{2}\right)^{-0.5}, v\left(u^{2}-v^{2}\right)^{-0.5} ; v\left(u^{2}-v^{2}\right)^{-0.5}, u\left(u^{2}-v^{2}\right)^{-0.5}\right]
$$

Now let us describe results of the author's study of eigenvalues of the symmetric matrices in the parastichic sequence (3.5). Each of these matrices $\left[\mathrm{F}_{n+1}, \mathrm{~F}_{n} ; \mathrm{F}_{n}, \mathrm{~F}_{n+1}\right]$ has two eigenvalues, which are equal to two Fibonacci numbers again: $\mathrm{F}_{n+2}$ and $\mathrm{F}_{n-1}$. One can noted that these eigenvalues are the sum and the difference of the Fibonacci components of the 
original hyperbolic number $\mathrm{F}_{n+1}+\mathrm{j} \mathrm{F}_{n}$ since $\mathrm{F}_{n+2}=\mathrm{F}_{n+1}+\mathrm{F}_{n}$ and $\mathrm{F}_{n-1}=\mathrm{F}_{n+1}-\mathrm{F}_{n}$. The ratio $\mathrm{F}_{n+2} / \mathrm{F}_{n-1}$ of such eigenvalues defines a new sequence (11) of Fibonacci ratios, which tend to $\varphi^{3}$ as $n$ increases:

$$
\mathrm{F}_{n+2} / \mathrm{F}_{n-1}: \quad 3 / 1,5 / 1,8 / 2,13 / 3,21 / 5,34 / 8,55 / 13, \ldots
$$

By analogy with expressions $(3.2,3.4,3.5)$ such pair of eigenvalues $\mathrm{F}_{n+2}$ and $\mathrm{F}_{n-1}$ can be considered as components of a new hyperbolic number $\mathrm{F}_{n+2}+\mathrm{j} \mathrm{F}_{n-1}$. In this case the sequence of ratios (3.9) is transformed into additive sequences $(3.10,3.11)$ reflecting linear notation of appropriate hyperbolic numbers and their matrix presentations:

$$
\begin{gathered}
\mathrm{F}_{n+2}+\mathrm{j} \mathrm{F}_{n-1}: \quad 3+\mathrm{j}, 5+\mathrm{j}, 8+\mathrm{j} 2,13+\mathrm{j} 3,21+\mathrm{j} 5,34+\mathrm{j} 8,55+\mathrm{j} 13, \ldots \\
\left|\begin{array}{c}
\mathrm{F}_{n+2}, \mathrm{~F}_{n-1} \\
\mathrm{~F}_{n-1}, \mathrm{~F}_{n+2}
\end{array}\right|:\left|\begin{array}{l}
3,1 \\
1,3
\end{array}\right|,\left|\begin{array}{l}
5,1 \\
1,5
\end{array}\right|,\left|\begin{array}{c}
8,2 \\
2,8
\end{array}\right|,\left|\begin{array}{c}
13,3 \\
3,13
\end{array}\right|,\left|\begin{array}{c}
21,5 \\
5,21
\end{array}\right| \ldots
\end{gathered}
$$

Each of symmetric matrices $\left[\mathrm{F}_{n+2}, \mathrm{~F}_{n-1} ; \mathrm{F}_{n-1}, \mathrm{~F}_{n+2}\right]$ of the sequence (3.11) has two eigenvalues, which are again equal to two Fibonacci numbers multiplied by a factor 2 (twice the Fibonacci numbers): $2 \mathrm{~F}_{n+1}$ and $2 \mathrm{~F}_{n}$. Ratios $2 \mathrm{~F}_{n+1} / 2 \mathrm{~F}_{n}$ of such eigenvalues form a sequence, which is identical to the initial parastichic sequence (3.2). Using the Binet's formula (3.1), all members of these sequences can be additionally expressed through the golden ratio $\varphi$ in integer powers. This procedure of analysis of the eigenvalues of new and new sequences of symmetric matrices, representing hyperbolic numbers by analogy with sequences $(3.4,3.5$, $3.10,3.11$ ), can be repeated as long as desired, obtaining a hierarchy of eigenvalues of the matrices based on Fibonacci numbers multiplied by a factor 2 at corresponding steps of the iterative procesure.

The following important point should be emphasized. In contrast to the traditional additive series of one-dimensional Fibonacci numbers, the author introduces an additive series of two-dimensional hyperbolic numbers and an additive series of (2*2)-matrices representing these numbers and defining an additional additive series of eigenvalues of these matrices $(3.4,3.5,3.10,3.11)$. As far as we know, such Fibonacci series of two-dimensional numbers have not been described in the literature by anyone, and therefore they can be considered new in the extensive subject matter of Fibonacci numbers and their applications (some of author's results of the study of additive series of 4-dimensional hyperbolic Fibonacci numbers will be presented below).

Similar results are obtained by considering the additive series of two-dimensional hyperbolic Lucas numbers and the additive series of their matrix representations, which determine the additive series of eigenvalues of these symmetric matrices (these results are been publishing in a separate article). Here one can remind that one-dimensional Lucas numbers form the series $\mathrm{L}_{n+2}=\mathrm{L}_{n}+\mathrm{L}_{n+1}: 2,1,3,4,7,11,18, \ldots$, which is also known in phyllotaxis laws [Jean, 2006]. A study of additive series of complex numbers, whose 
12

components are Fibonacci numbers, and of their ordinary representations by non-symmetric matrices gives also interesting additive series of their eigenvalues but in form of complex numbers.

It should be noted that the study of the eigenvalues of symmetric matrices has special meaning due to the fact that in the theory of oscillations symmetric matrices are matrix representations of oscillatory systems with many degrees of freedom. Moreover, the eigenvalues of such a matrix determine the resonant frequencies of the corresponding oscillatory system. The described results on the properties of inherited phyllotaxis phenomena with their Fibonacci ratios, represented by symmetric matrices and their matrix eigenvalues, are important, in particular, for the concept of multi-resonance genetics, which connects structural features of molecular-genetic systems with resonances of oscillatory systems [Petoukhov, 2016].

\section{Fibonacci sequences of $2^{n}$-dimensional hyperbolic numbers}

This Section continues the theme of additive series of hyperbolic numbers, coordinates of which are Fibonacci numbers. Now we turn to algebraic extensions of hyperbolic numbers in forms of $2^{n}$-dimensional hyperbolic numbers. Let us consider an additive sequence (4.1) of 4-dimensional hyperbolic numbers $\mathrm{F}_{n+3} \mathrm{e}_{0}+\mathrm{F}_{n+2} \mathrm{e}_{1}+\mathrm{F}_{n+1} \mathrm{e}_{2}+\mathrm{F}_{n} \mathrm{e}_{3}$ with Fibonacci coordinates from (Table 3.1). In this sequence, each member is equal to the sum of two previous members:

$$
3 \mathrm{e}_{0}+2 \mathrm{e}_{1}+1 \mathrm{e}_{2}+1 \mathrm{e}_{3} ; 5 \mathrm{e}_{0}+3 \mathrm{e}_{1}+2 \mathrm{e}_{2}+1 \mathrm{e}_{3} ; 8 \mathrm{e}_{0}+5 \mathrm{e}_{1}+3 \mathrm{e}_{2}+2 \mathrm{e}_{3} ; 13 \mathrm{e}_{0}+8 \mathrm{e}_{1}+5 \mathrm{e}_{2}+3 \mathrm{e}_{3} ; \ldots
$$

A corresponding matrix representation of each member from (4.1) has 4 eigenvalues, which can be considered again as coordinates of a new 4-dimensional hyperbolic number. The author reveals that these new 4-dimensional hyperbolic numbers form a new additive sequence (4.2):

$$
1 e_{0}+1 e_{1}+3 e_{2}+7 e_{3} ; 1 e_{0}+3 e_{1}+5 e_{2}+11 e_{3} ; 2 e_{0}+4 e_{1}+8 e_{2}+18 e_{3} ; 3 e_{0}+7 e_{1}+13 e_{2}+29 e_{3} ; \ldots
$$

The sequence (4.2) combines Fibonacci and Lucas sequences in the following sense. In its 4-dimensional hyperbolic numbers, coordinates of basis elements $\mathrm{e}_{0}$ and $\mathrm{e}_{2}$ are Fibonacci numbers and coordinates of basis elements $e_{1}$ and $e_{3}$ are Lucas numbers: 3, 1, 4, 7, 11, 18, 29, ... Such aggregation of Fibonacci and Lucas numbers resembles a phyllotaxis-like locations of amino acid residues in the helices of polypeptides for various molecular chains - 11/3, 18/5, 29/8, 47/13; here fraction numerators are Lucas numbers and fraction denominators are Fibonacci numbers. These bio-molecular phenomena of polypeptides configurations are described in the fundamental book [Frey-Wissling, Muhlethaler, 1965].

A matrix representation of each member of the sequence (4.2) has 4 eigenvalues, which can be considered again as coordinates of a new 4-dimensional hyperbolic number. These 4-dimensional hyperbolic numbers form a new additive sequence (4.3): 
$-8 \mathrm{e}_{0}-4 \mathrm{e}_{1}+4 \mathrm{e}_{2}+12 \mathrm{e}_{3} ;-12 \mathrm{e}_{0}-8 \mathrm{e}_{1}+4 \mathrm{e}_{2}+20 \mathrm{e}_{3} ;-20 \mathrm{e}_{0}-12 \mathrm{e}_{1}+8 \mathrm{e}_{2}+32 \mathrm{e}_{3} ;-32 \mathrm{e}_{0}-20 \mathrm{e}_{1}+12 \mathrm{e}_{2}+32 \mathrm{e}_{3} ; .$.

Comparing sequences (4.1) and (4.3) reveals that a set of coordinates of each member of the sequence (4.3) repeats - with a factor 4 - a set of coordinates of the corresponding member of the sequence (4.1) with accuracy up to signs and a cyclic permutation of coordinates. For example, the first member of (4.1) contains coordinates 3, 2, 1, 1 and the first member of (4.3) contains coordinates $-4 * 2,-4 * 1,4 * 1,4 * 3$. This procedure of calculating repeating additive sequences of 4-dimensional hyperbolic numbers associated with Fibonacci and Lucas numbers can be repeated as long as desired. Similar results are received for additive sequences of $2^{n}$-dimensional hyperbolic numbers with Fibonacci coordinates in cases $n=3,4, \ldots$.

\section{Hyperbolic numbers and the Weber-Fechner law}

It is profitable for an organism, which is a single whole, to have the same typical algorithms at different levels of its functioning for a mutual optimal coordination of its parts. By this reason we study possibilities to simulate differentinnate phenomena on the general basis of hyperbolic numbers and its algebraic extensions. This Section is devoted to the main psychophysical law by Weber-Fechner and its structural connection with phyllotaxis laws through hyperbolic numbers. The innate Weber-Fechner law states that the intensity of the perception is proportional to the logarithm of stimulus intensity; it is expressed by the equation (5.1):

$$
\mathrm{p}=\mathrm{k}^{*} \ln \left(\mathrm{x} / \mathrm{x}_{0}\right)=\mathrm{k}^{*}\left\{\ln (\mathrm{x})-\ln \left(\mathrm{x}_{0}\right)\right\}
$$

where $\mathrm{p}$ - the intensity of perception, $\mathrm{x}$ - stimulus intensity, $\mathrm{x}_{0}$ - threshold stimulus, ln - natural logarithm, $\mathrm{k}$ - a weight factor. It is known that different types of inherited sensory perception are subordinated to this law: sight, hearing, smell, touch, taste, etc. Because of this law, the power of sound in physics and engineering technologies is measured on a logarithmic scale in decibels.

One can suppose that the innate Weber-Fechner law is the law especially for nervous system. But it is not so since its meaning is much wider because it holds true in many kinds of lower organisms without a nervous system in them: "this law is applicable to chemotropical, helio-tropical and geo-tropical movements of bacteria, fungi and antherozoids of ferns, mosses and phanerogams ... . The Weber-Fechner law, therefore, is not the law of the nervous system and its centers, but the law of protoplasm in general and its ability to respond to stimuli" [Shults, 1916, p.126]

Let us show that hyperbolic numbers are related to the Weber-Fechner law, which is based on the natural logarithm (5.1). Historically the natural logarithm was formerly termed the hyperbolic logarithm, as it corresponds to the area under a hyperbola [Klein, 2004; Shervatov, 1954]. History of hyperbolic logarithms is described for example in the book [Klein, 2004]. As known, the natural logarithm can be defined for any positive real number " $a$ " as the area under the hyperbola $\mathrm{y}=1 / \mathrm{x}$ from 1 to $a$ (Fig. 5.1, left). It means that two points of the hyperbola with their coordinates $(\mathrm{x}, 1 / \mathrm{x})$ and $\left(\mathrm{x}_{0}, 1 / \mathrm{x}_{0}\right)$, where $\mathrm{x}>1$ and $\mathrm{x}_{0}>1$, 
14

define values of natural logarithms $\ln (\mathrm{x})$ and $\ln \left(\mathrm{x}_{0}\right)$. Subtraction $\ln (\mathrm{x})-\ln \left(\mathrm{x}_{0}\right)=\ln \left(\mathrm{x} / \mathrm{x}_{0}\right)$ expresses the intensity of perception $\mathrm{p}$ in the expression (5.1) of the Weber-Fechner law (Fig. 5.1, right).
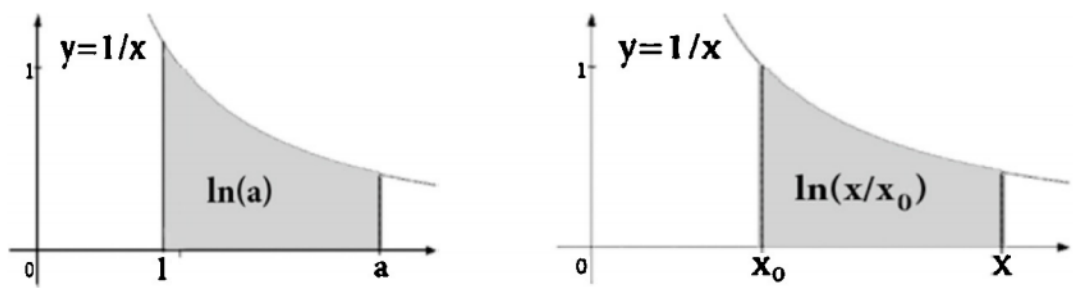

Fig. 5.1. Natural logarithm as the area under the hyperbola $y=1 / x$. Left: $\ln (a)$ is equal to the area under the hyperbola from 1 to a. Right: $\ln \left(\mathrm{x}_{\mathrm{x}} \mathrm{x}_{0}\right)$ is equal to the area under the hyperbola from $\mathrm{x}_{0}$ to $\mathrm{x}$.

A plane of the hyperbola $y=1 / x$ can be naturally considered as the hyperbolic plane where points $(\mathrm{x}, 1 / \mathrm{x})$ and $\left(\mathrm{x}_{0}, 1 / \mathrm{x}_{0}\right)$ on this hyperbola are defined as hyperbolic numbers $\mathrm{x}+\mathrm{j} 1 / \mathrm{x}$ and $\mathrm{x}_{0}+\mathrm{j} 1 / \mathrm{x}_{0}$. From the standpoint of the expression (5.1) of the Weber-Fechner law, any transformation of a stimulus intensity $\mathrm{x}\left(\mathrm{x}>\mathrm{x}_{0}\right)$ into a new stimulus intensity $\mathrm{x}_{2}\left(\mathrm{x}_{2}>\mathrm{x}_{0}\right)$ corresponds to the case that the hyperbolic number $x+j 1 / x$ is transformed into a new hyperbolic number $\mathrm{x}_{2}+\mathrm{j} 1 / \mathrm{x}_{2}$ on the same hyperbola $\mathrm{y}=1 / \mathrm{x}$ by means of multiplication of the first hyperbolic number with another hyperbolic number $u+j v$ that is $(x+j 1 / x)(u+j v)=x_{2}+j 1 / x_{2}$ where $\mathrm{u}=\left(\mathrm{x}_{2}{ }^{2} \mathrm{x}^{3}-\mathrm{x}\right) /\left(\mathrm{x}_{2}\left(\mathrm{x}^{4}-1\right)\right), \mathrm{v}=\mathrm{x}\left(\mathrm{x}_{2}^{2}-\mathrm{x}^{2}\right) /\left(\mathrm{x}_{2}\left(1-\mathrm{x}^{4}\right)\right)$.

This analysis gives evidences that our sensory perception obeys the same structural principles as morphogenesis with its phyllotaxis laws and that these principles can be effectively modelling on the basis of hyperbolic numbers.

\section{The alphabets of orthogonal vector bases associated with basis units of $2^{n}$-dimensional hyperbolic numbers}

Let us remind the essence of the eigenvalues and eigenvectors by means of the matrix A on Fig. 6.1, which acts on vectors $[\mathrm{x}, \mathrm{y}]$. In this case almost any vector is transformed into a new vector $[\mathrm{x}, \mathrm{y}]^{*} \mathrm{~A}$ with changing its direction. The exceptions are those vectors $[\mathrm{x}, \mathrm{y}]$, which belong to two orthogonal dotted lines and are called "eigenvectors" of the matrix A; they conserve their direction under action of the matrix A, but their lengths are scaled with factors $\lambda_{\mathrm{i}}$, which are called "eigenvalues" of the matrix A (each eigenvalue corresponds to its own direction of eigenvectors). 

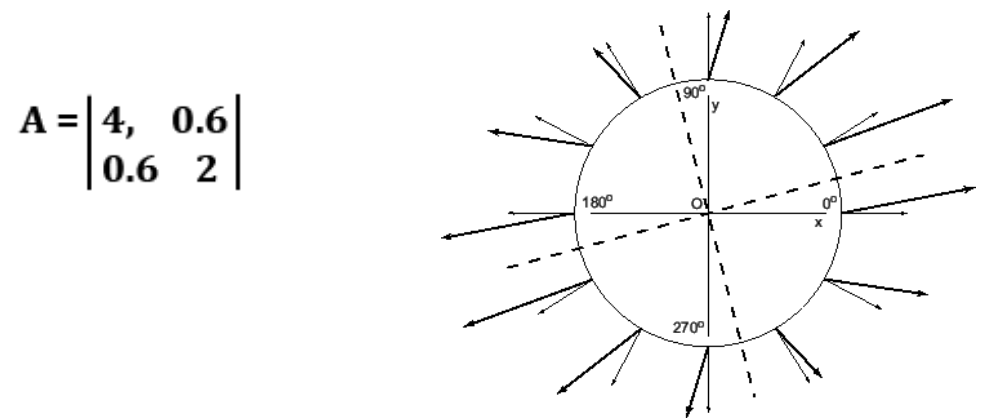

Fig. 6.1. Illustration of actions of the matrix A on vectors [x, y] (from [Zharov, 2002])

Each basis unit of $2^{n}$-hyperbolic numbers is represented by a corresponding symmetric $\left(2^{\mathrm{n} *} 2^{\mathrm{n}}\right)$-matrix, which is an orthogonal matrix and has its own set of orthogonal eigenvectors. This orthogonal set is a corresponding vector basis of $2^{n}$-dimensional space. For example in the case of any 2-dimensional hyperbolic number $a^{*} \mathrm{j}_{0}+b^{*} \mathrm{j}_{1}$ (Fig. 2.4) its real component $a \mathrm{j}_{0}$ is presented by the matrix $a^{*}[1,0 ; 01]$, which has two orthogonal eigenvectors $[1,0]$ and $[0,1]$ independently on value of the coefficient $a(a \neq 0)$. This pair of eigenvectors defines the first vector basis of the 2-dimensional space of existance of hyperbolic numbers. The imaginary term $b \mathrm{j}_{1}$ is presented by the matrix $b^{*}[0,1 ; 1,0]$ (Fig. 2.4), which has another pair of orthogonal eigenvectors $\left[-2^{-0.5}, 2^{-0.5}\right],\left[2^{-0.5}, 2^{-0.5}\right]$ independently on value of the coefficient $b(b \neq 0)$. This pair of eigenvectors defines the second vector basis of the considered 2-dimensional space. In other words, the pairs of eigenvectors are determined only by basis units $\mathrm{j}_{0}$ and $\mathrm{j}_{1}$. These two pairs of eigenvector bases can be considered as a two-term vector alphabet of basis units of hyperbolic numbers in case of 2-dimensional space.

A similar situation is true for cases of other $2^{n}$-dimensional hyperbolic numbers and eigenvectors of their matrix representations. For example, in the case of 4-dimensional hyperbolic numbers $a \mathrm{e}_{0}+b^{*} \mathrm{e}_{1}+c^{*} \mathrm{e}_{2}+d^{*} \mathrm{e}_{3}$, matrix representations of their basis units (see Fig. 2.5) have the following eigenvectors:

- The (4*4)-matrix $[1,0,0,0 ; 0,1,0,0 ; 0,0,1,0 ; 0,0,0,1]$ representing the real unit $\mathrm{e}_{0}$ has 4 eigenvectors $[1,0,0,0],[0,1,0,0],[0,0,1,0],[0,0,0,1]$;

- The (4*4)-matrix $[0,1,00 ; 1,0,0,0 ; 0,0,0,1 ; 0,0,1,0]$ representing the first

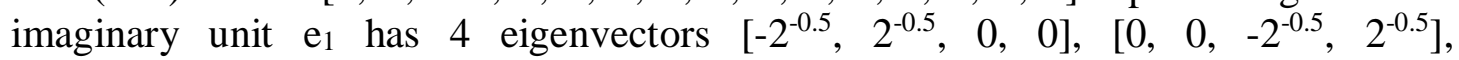
$\left[0,0,2^{-0.5}, 2^{-0.5}\right],\left[2^{-0.5}, 2^{-0.5}, 0,0\right]$;

- The (4*4)-matrix $[0,0,1,0 ; 0,0,0,1 ; 1,0,0,0 ; 0,1,0,0]$ representing the second imaginary unit $\mathrm{e}_{2}$ has 4 eigenvectors $\left[\begin{array}{llll}-2^{-0.5}, 0, & 2^{-0.5}, 0\end{array}\right],\left[\begin{array}{lll}0,-0.5 & 0, & -2^{-0.5}\end{array}\right]$, $\left[0,2^{-0.5}, 0,2^{-0.5}\right],\left[-2^{-0.5}, 0,-2^{-0.5}, 0\right]$;

- The (4*4)-matrix $[0,0,0,1 ; 0,0,1,0 ; 0,1,0,0 ; 1,0,0,0]$ representing the third imaginary unit $\mathrm{e}_{3}$ has 4 eigenvectors $\left[0,-2^{-0.5}, 2^{-0.5}, 0\right],\left[2^{-0.5}, 0,0,-2^{-0.5}\right]$, $\left[2^{-0.5}, 0,0,2^{-0.5}\right],\left[0,2^{-0.5}, 2^{-0.5}, 0\right]$.

Correspondingly in the case of 4-dimensional hyperbolic numbers and their space, the 4-term eigenvector alphabet of their 4 basis units exists. In a general case of $2^{n}$-dimensional hyperbolic numbers, the $2^{n}$-term eigenvector alphabet of their $2^{n}$ basis units exists. Each member of such alphabet is a set of $2^{n}$ orthogonal vectors. The author briefly calls such alphabets of eigenvector bases of matrix representations of basis units of $2^{n}$-dimensional hyperbolic numbers as «hyperbolic eigenvector alphabets» or simply «hyper-alphabets». Here the prefix "hyper" is the beginning of the word "hyperbolic" and its use is additionally 
16

justified by the fact that each member of such hyper-alphabet contains in itself another alphabet of a set of eigenvectors of the corresponding basis unit.

Any transformation of one such eigenvector basis into another (that is a transformation of one member of a hyper-alphabet into another) is provided by means of an orthogonal matrix (orthogonal operator), that is, a real unitary matrix (previously, the structural connection of DNA alphabets with orthogonal matrices was shown by the author in [Petoukhov, 2018a]; unitary operators play a great role in quantum mechanics and quantum computing; for example, all calculations in quantum computers are based on unitary operators). Orthogonal operators preserve the space metric and define transformations of proper and improper rotations. Any sequence of basis units (or their sums) of $2^{n}$-dimensional hyperbolic numbers corresponds to a certain sequence of eigenvector bases of these units, and also to a sequence of orthogonal matrices transforming successively these bases. Such algebraic sequences can be used for transmitting information. Taking into account some results of his previous published studies, the author supposes that genetic sequences are related with such algebraic sequences.

Moreover, the author puts forward the hypothesis that alphabets of eigenvectors of matrix representations of basis units of $2^{n}$-dimensional hyperbolic numbers play a key role in transmitting biological information and that they can be considered as a foundation of coding information at different levels of biological organization. The corresponding languages using such alphabets define many inherited phenomenological structures in biology including molecular-genetic structures.

As known, the principle of transmitting information in the form of certain texts composed on the basis of certain "alphabets" is widely used in living organisms: genetic information is recorded in DNA molecules in the form of texts based on the DNA alphabet; music is a sequence of sound frequencies of one or another musical scale (that is, the "alphabet" of note sound frequencies of one octave); literary texts are written on the basis of literary alphabets, etc. The author believes that various alphabets and texts in these bioinformational fields can be effectively modeled and studied on the basis of the presented hidden algebraic alphabets as their joint algebraic foundation. This approach is connected with the theme of a «grammar of biology», which term was introduced by E.Chargaff in the title of his article on DNA peculiarities «Preface to a Grammar of Biology» [Chargaff, 1971] (see also the book [Yamagishi, 2017]).

Since alphabets are used as foundations of corresponding languages, each algebraic hyper-alphabet in $2^{n}$-dimensional spaces with a concrete number $n$ can be considered as a foundation of a corresponding algebraic language. From this point of view, many such algebraic languages using these hyper-alphabets exist in biology.

\section{Quint ratios in DNA parameters and musical harmony}

As known, thoughts about the key significance of musical harmony in the organization of the world exist from ancient time. For example, one can quote here a classical work of Chinese literature "Spring and Autumn" by $\mathrm{Lu} \mathrm{Bu}$ We about the fundamental role of music and numbers 3 and 2 as numbers of Heaven and Earth: "The origins of music lie far back in the past. Music arises from Measure and is rooted in the great Oneness. ... Music is founded 
on the harmony between Heaven and Earth" (this citation is taken from the book [Hesse, 2002]. In Ancient China the ratio 3/2, traditionally termed as the quint ratio (or the pure perfect fifth), was used as the fundament of quint music scales. After Ancient Chinese, Pythagoreans also considered numbers 2 and 3 as the female and male numbers (or Yin and Yang numbers), which can give birth to new musical tones in their interconnection. Ancient Greeks attached an extraordinary significance to search of the quint 3:2 in natural systems because of their thoughts about musical harmony in the organization of the world. For example, Archimedes considered as the best result of his life a detection of the quint $3 / 2$ between volumes and surfaces of a cylinder and a sphere entered in it.

Science has been dealing with the physiological mechanisms of music perception for a long time [Weinberger, 2004]. There is no specialized center of music in the human brain, a sense of love for music can be considered dispersed throughout the body, similar to the dispersion of genetic DNA molecules throughout all of its cells. More than 30 thousand years ago, long before the advent of arithmetic, our ancestors already played stone flutes and bone harps. For example, the bone flute found in France is at least 32 thousand years old. The enjoyment of music is usually explained by the fact that it gives rise to emotions and feelings. Aristotle tried to understand how rhythms and melodies, being only sounds, resemble states of mind. Available data indicate that our affinity for music and musical creativity is biological in nature and the sense of musical harmony is based on innate mechanisms. Therefore, one should look for a connection between the genetic system and musical harmony.

For Europeans the idea of musical harmony is basically connected with the name Pythagoras. The Pythagorean musical scales, which are based on the quint ratio 3/2, played the main role in the Pythagorean's doctrine about a cosmic meaning of musical harmony. Fig. 7.1 shows the known interconnection of sound frequencies of notes of Pythagorean 7-stages scale (a heptatonic scale) on the basis of the ratio $3 / 2$ when notes are spaced in the appropriate octaves.

\begin{tabular}{|l|l|l|l|l|l|l|}
\hline fa $(\mathrm{F})$ & do $(\mathrm{C})$ & sol $(\mathrm{G})$ & re $\left(\mathrm{D}^{1}\right)$ & la $\left(\mathrm{A}^{1}\right)$ & $\mathrm{mi}\left(\mathrm{E}^{2}\right)$ & si $\left(\mathrm{B}^{2}\right)$ \\
\hline 87 & 130 & 196 & 293 & 440 & 660 & 990 \\
\hline $\mathbf{( 3 / 2})^{-\mathbf{3}}$ & $\mathbf{( 3 / 2})^{-\mathbf{2}}$ & $\mathbf{( 3 / 2})^{-\mathbf{1}}$ & $\mathbf{( 3 / 2})^{\mathbf{0}}$ & $\mathbf{( 3 / 2})^{\mathbf{1}}$ & $\mathbf{( 3 / 2})^{\mathbf{2}}$ & $\mathbf{( 3 / 2})^{\mathbf{3}}$ \\
\hline
\end{tabular}

Fig. 7.1. The quint sequence of the 7 notes of the Pythagorean musical scale is presented. The upper row shows the notes. The second row shows their frequencies. The third row shows the ratios between the frequencies of these notes to the frequency $293 \mathrm{~Hz}$ of the note re $\left(\mathrm{D}^{1}\right)$. The designation of notes is given on Helmholtz system. Values of frequencies are approximated to integers.

Pythagoras created the mathematical foundations of ancient Greek music, borrowing in a certian degree some ancient knowledge on musical harmony. His theory used the discovery that the frequency of a vibrating string is inversely proportional to its length and that musical consonances can be represented by the ratios of small integer numbers, first of all the octave ratio $2: 1$ and the quint ratio $3: 2$. These ideas became the basic fundamental ones of all music theory from antiquity to even modern times. For most Europeans from antiquity, quint scales in music are connected with this Pythagorean mathematical theory of musical harmony and with divisions of vibrating strings in the quint ratio $3: 2$. 
18

In a general case, the Pythagorean scale is any scale, which can be constructed from only quint ratios 3:2 and octaves 2:1 [Sethares, 2005, p. 163]. One of known Pythagorean scales is a pentatonical scale, which is a five-stages music scale, all the sounds of which can be arranged in quint ratios. Its example is the set of the following 5 notes with their sound frequencies from Fig. 7.1: $\operatorname{do}(C)-\operatorname{sol}(G)-r e\left(D^{1}\right)-1 a\left(A^{1}\right)-m i\left(E^{2}\right)$ or respectedly 130-196-293440-660 Hz. Other examples of Pythagorean scales are tetratonic and tritonic scales, which are correspondingly 4-stages and 3-stages music scales, all the sounds of which can be arranged by the quint ratio, for instance, 130-196-293-440 Hz for the tetratonic scale and $130-196-293 \mathrm{~Hz}$ for the tritonic scale.

The historical fact is that these Pythagorean musical scales on the basis of the quint ratio were used by different civilisations around the world long before Pythagoras without knowledge of any mathematical laws [Apel, 1969; Day-O'Connell, 2007; Christidis, Arapopoulou, Christi, 2007; Olsen, Sheehy, 1998; Todd Titon, 1996]. For example, the pentatonical scale is the foundation of traditional music of the Chinese, Vietnamese, Mongols, Turkic peoples (Bashkirs, Tatars, Chuvashes, etc.), the Inca Empire and the peoples of the South Andes in general. Pentatonics is also found in European musical folklore and in the oldest layers of the Russian folk song (especially in the so-called calendar ritual songs). Tetratonic music was noted as common in Polynesia and Melanesia. Tetratonic scales were known for example among the Plains Indians, the Arapaho, Blackfoot, Crow, Omaha, Kiowa, Pawnee, Sioux, some Plateau tribes, the Creek Indians, and in the Great Basin region among the Washo, Ute, Paiute, and Shoshone. In the Southwest, the Navajo people also largely used the pentatonic and tetratonic, occasionally also tritonic scales. Tetratonic, as well as tritonic scales, were commonly used by the tribal peoples of India, such as the Juang and Bhuyan of Orissa state [Sudhibhushan Bhattacharya, 1968]. Tetratonic scales are generally associated with prehistoric music [Baines, 1991].

G.Leibniz declared that music is arithmetic of soul, which computes without being aware of it. But what is there in living organisms that determines the special attraction of musical scales on the basis of the quint ratio $3 / 2$ for representatives of various civilizations and epochs? A possible answer lies in the structural features of DNA molecules that are carriers of genetic information in humans and other living organisms. The author has paid attention to the fact that the parametric structure of DNA molecules is connected in many ways with the quint ratio $3 / 2$ and with numbers 3 and 2 at various levels of their parametric organization [Petoukhov, 2008; Petoukhov, He, 2010]. Let us briefly say now about this relation between the musical harmony and structures of genetic molecules.

Molecules of heredity - DNA and RNA - contain sequences of 4 "letters" or nucleobases: adenine $(A)$, cytosine $(C)$, guanine $(\mathrm{G})$, thymine $(\mathrm{T})$ (or uracil $\mathrm{U}$ in RNA). Letters A-T(U) and $\mathrm{C}-\mathrm{G}$ form complementary pairs with 2 and 3 hydrogen bonds in them, respectively. From the standpoint of its sequence of two and three hydrogen bonds, each DNA molecule is a long chain of numbers 2 and 3 of a type 32232332 ....

The genetic code encodes sequences of 20 amino acids in proteins by means of 64 triplets (three-letter words) that represent all possible combinations of these four letters (ATC, TTA, ...). Since $A=T=2, C=G=3$, each triplet has a numeric representation as a product of number of hydrogen bonds of its constituent letters. For example, the triplet ACT is represented by number $2 * 3 * 2=12$. Each of 64 triplets is represented by one of such numbers of hydrogen bonds $2^{3}=8,2^{2} * 3=12,2^{*} 3^{2}=18,3^{3}=27$, the pairwise relations between which are 
equal to the quint $3 / 2$ in varying integer degrees (by analogy with music tetratonic scales), for example, $27 / 8=(3 / 2)^{3}, 18 / 8=(3 / 2)^{2}$, etc.

Under considering pairs of adjacent triplets, then DNA molecule appears as a quint sequence of 7 kinds of numbers of hydrogen bonds with the following numeric representation: $2^{6}=64,2^{5 * 3}=96,2^{4 *} 3^{2}=144, \quad 2^{3 * 3^{3}}=216,2^{2 *} 3^{4}=324, \quad 2^{*} 3^{5}=486, \quad 3^{6}=729$. Pairwise ratios in this series of numbers are equal to the quint $3 / 2$ in the same powers as in the Pythagoras 7-stage scale in Fig. 7.1. If, for example, the frequency of $87 \mathrm{~Hz}$ of the note " $F "$ is compared with the first number 64 of this series, then all other numbers of this series will correspond precisely to the other frequencies of the Pythagoras scale. Then any sequence of triplets (eg, insulin gene GGC-ATC-GTT-GAA-CAG-TGT- ...) can be associated uniquely with a sequence of notes of Pythagoras 7-stages scale (figuratively speaking, we have "music of genes in the Pythagoras scale").

Accordingly, each DNA molecule as a chain of hydrogen bonds is characterized by its own sequences of the quint $3 / 2$ in different integer degrees. By analogy with quint musical scales, depending on the chosen lengths of nucleobase fragments of DNA, we have - on the basis of considered hydrogen bonds - various systems for transmitting information signals with quint-power relations between signals.

The quint ratios are realized in DNA not only for the hydrogen bonds of complementary nucleobases, but also for several other parameters, such as sums of atoms in the rings of purines and pyrimidines (numbers 9 and 6 with their ratio 3/2), or sums of protons in the rings of complementary nitrogenous bases (numbers 60 and 40 with their ratio 3/2), and others. Chains of these parameters in DNA form their own sequences of quint ratios, which are similar to sequences of note frequencies in quint scales of music. In other words, Nature created DNA as a plexus of various sequences of quint ratios ("a quint polyphony of DNA"). The harmony of the parametric organization of the genetic system is akin to the musical harmony of the Pythagorean scales.

As it was reminded above, over the centuries from Ancient China to antiquity, the numbers 2 and 3 were considered respectively as female and male numbers (that is as Yin and Yang numbers) forming the important pair. The author proposes their consideration not as separate one-dimensional numbers but as two separate parts of two-dimensional number. Mathematics knows 3 main kinds of two-dimensional numbers: complex numbers, hyperbolic (or double) numbers and dual numbers [Kantor, Solodovnikov, 1989]. Taking into account a set of our results on relations of genetic system and inherited physiological phenomena with hyperbolic numbers, we choice namely hyperbolic numbers for a presentation of these historically known numbers 3 and 2 as two interrelated parts of single two-dimensional number $\mathrm{G}_{2}=3+2 \mathrm{j}$, where $\mathrm{j}$ is imaginary unit with its feature $\mathrm{j}^{2}=+1$; the index 2 refers 2-dimensionality of the number $\mathrm{G}_{2}$. This hyperbolic number can be expressed as a point or a vector on a hyperbolic plane with Cartesian coordinates, in which the axis of abcissus is considered the axis of Yang-numbers, and the axis of ordinates is considered the axis of Yin-numbers. Fig. 7.2 shows this coordinate system and also the matrix form of presentation of hyperbolic numbers with its decomposition into 2 sparse matrices playing the role of real and imaginary basis units of hyperbolic numbers. This matrix [3, 2; 2, 3] is conditionally termed "quint matrix" since its components 3 and 2 give the ratio 3/2. (The same quint matrix $[3,2 ; 2,3]$ appears under a consideration of DNA alphabet C, A, T, G and its three binary sub-alphabets [Petoukhov, 2008, Chapter 2; Petoukhov, He, 2010, Chapter 4]. 


$$
\left.\underset{3 \text { Yang }}{\longrightarrow} \mathrm{G}_{2}=\left|\begin{array}{l}
3,2 \\
2,3
\end{array}\right|=3 *\left|\begin{array}{l}
1,0 \\
0,1
\end{array}\right|+2 * \mid \begin{array}{l}
0,1 \\
1,0
\end{array}\right) ; \quad \begin{array}{|l|l|l|}
* & 1 & \mathrm{j}_{1} \\
\hline 1 & 1 & \mathrm{j}_{1} \\
\hline \mathrm{j}_{1} & \mathrm{j}_{1} & 1 \\
\hline
\end{array}
$$

Fig. 7.2. The graphical and matrix presentation of 2-dimensional hyperbolic number $\mathrm{G}_{2}=3+2 \mathrm{j}_{1}$ (by analogy with Fig. 2.4). The first sparse matrix $[1,0 ; 0,1]$ is the identity matrix, the second sparse matrix $[0,1 ; 1,0]$ presents imaginary unit $\mathrm{j}_{1}$ having the property $[0,1 ; 1,0]^{2}=[1,0 ; 0,1]$. The multiplication table of these sparse matrices, where 1 refers the matrix $[1,0 ; 0,1]$, is also shown at right.

\section{Applications of algebras of $2^{n}$-dimensional hyperbolic numbers in musicology}

This Section is devoted to relations of Pythagorean musical scales with $2^{n}$-dimensional hyperbolic numbers and also to a possibility of using hyper-alphabets of eigenvectors of matrix representations of their basis units for progress in mathematical musicology (see mathematical explanations above in Sections 2 and 6). It should be emphasized an important differency between a traditional using in musicology one-dimensional numbers (which provide a comparison numeric analysis of sound frequencies of various notes) and the proposed using the multi-dimensional numbers. In the case of using the described multidimensional numbers, cardinally new personages come into play: matrix representations of these numbers with orthogonal systems of eigenvectors of their basis units, sets of which form hyper-alphabets described above in Section 6. These new personages allow significantly encreasing analytical possibilities in musicology by means of those mathematical tools, which are effectively used in many scientific and technology fields.

Let us show now that exponentiation of the quint matrix $[3,2 ; 2,3]$ into tensor powers $n=2,3,4, \ldots$ generate $2^{n}$-dimensional hyperbolic numbers, whose components form sets similar to the sets of sound frequencies of the Pythagorean quint scales in the following sense: ratios between any pair of their components are equal to the ratio $3 / 2$ in integer powers. The tensor product of matrices [Bellman, 1960] is widely applied in mathematics, physics, informatics, etc. It is used for algorithmic generation of higher dimensional spaces on the basis of spaces with smaller dimensions. By definition, the tensor product of two square matrices $\mathrm{V}$ and $\mathrm{W}$ of the orders $m$ and $n$ respectively is the matrix $\mathrm{Q}=\mathrm{V} \otimes \mathrm{W}=$ $\left\|\mathrm{v}_{\mathrm{ij}} * \mathrm{~W}\right\|$ with the order $m * n$. For example, the second tensor power of the initial (2*2)-matrix $[3,2 ; 2,3]^{(2)}$ gives the $(4 * 4)$-matrix $[9,6,6,4 ; 6,9,4,6 ; 6,4,9,6 ; 4,6,6,9]$, representing the 4-dimensional hyperbolic number $9 \mathrm{e}_{0}+6 \mathrm{e}_{1}+6 \mathrm{e}_{2}+4 \mathrm{e}_{3}$ where $\mathrm{e}_{0}, \mathrm{e}_{1}, \mathrm{e}_{2}, \mathrm{e}_{3}$ are basis units from Fig. 2.5. The set of components of this hyperbolic number consists of numbers $4,6,9$ with the following ratios between them: $9 / 6=3 / 2,9 / 4=(3 / 2)^{2}, 6 / 4=3 / 2$. The same ratios characterize the above mentioned tritonic musical scale 130-196-293 Hz: $293 / 196=3 / 2$, $293 / 130=(3 / 2)^{2}, 196 / 130=3 / 2$.

The third tensor power of $(2 * 2)$-matrix $[3,2 ; 2,3]^{(3)}$ gives an appropriate $(8 * 8)$-matrix representing the 8 -dimensional hyperbolic number $27+18 s_{1}+18 s_{2}+12 s_{3}+18 s_{4}+12 s_{5}+12 s_{6}+8 s_{7}$, where $s_{1}, s_{2}, \ldots, s_{7}$ are imaginary units. The set of components of this hyperbolic number consists of numbers $8,12,18,27$; pairwise ratios between them are identical to pairwise ratios between sound frequencies in the above mentioned Pythagorean tetratonic scale 130-196-293-440 Hz. By analogy the sixth tensor power of (2*2)-matrix $[3,2 ; 2,3]^{(6)}$ leads to 
64-dimensional hyperbolic number, whose components form the set with the same values of pairwise ratios as in Pythagorean 7-stages scale in Fig. 7.1.

Taking into account the described relations of Pythagorean musical scales with some $2^{n}$-dimensional hyperbolic numbers, the author proposes using $2^{n}$-dimensional hyperbolic numbers joinly with their hyper-alphabets of eigenvectors their basis units (see above Section 6) as a new mathematical tool in musicology for a possible revealing hidden regularities in products of musical creativity. The speech is that each of Pythagorean k-stages scales $(\mathrm{k}=2$, $3,4,5,6,7, \ldots)$ can be formally connected with an appropriate multi-dimensional hyperbolic number and its matrix representation. In other words, the author proposes to use mutual matching the members of the musical scale and the members of the hyper-alphabets of eigenvectors described above in Section 6.

For example, the above mentioned Pythagorean tetratonic scale with sound frequencies of its notes 130-196-293-440 Hz can be formally expressed by a matrix form of 4-dimensional hyperbolic number $130 \mathrm{e}_{0}+196 \mathrm{e}_{1}+293 \mathrm{e}_{2}+440 \mathrm{e}_{3}$ where $\mathrm{e}_{0}, \mathrm{e}_{1}, \mathrm{e}_{2}$ and $\mathrm{e}_{3}$ are basis units in their matrix representations from Fig. 2.5. In such case, musical notes of this Pythagorean scale have the following presentations using separate basis units of hyperbolic numbers:

- the note $\operatorname{do}(\mathrm{C})$ with its frequency $130 \mathrm{~Hz}$ is represented by the square matrix $130 \mathrm{e}_{0}$ simultaneously with its 4 eigenvectors and corresponding eigenvalues $\lambda_{\mathrm{i}}$ : $[1,0,0,0]$, $\lambda_{0}=130 ;[0,1,0,0], \lambda_{1}=130 ;[0,0,1,0], \lambda_{2}=130 ;[0,0,0,1], \lambda_{3}=130$;

- the note $\operatorname{sol}(\mathrm{G})$ with its frequency $196 \mathrm{~Hz}$ is represented by the square matrix $196 \mathrm{e}_{1}$ simultaneously with its 4 eigenvectors and their eigenvalues $\lambda_{\mathrm{i}}$ : [-0.7071, 0.7071, 0 , $0], \lambda_{0}=-196 ;[0,0,-0.7071,0.7071], \lambda_{1}=-196 ;[0,0,0.7071,0.7071], \lambda_{2}=196$; $[0.7071,0.7071,0,0], \lambda_{3}=196$;

- the note re $\left(D^{1}\right)$ with its frequency $293 \mathrm{~Hz}$ is represented by the square matrix $293 \mathrm{e}_{2}$ simultaneously with its 4 eigenvectors and 4 eigenvalues $\lambda_{\mathrm{i}}$ : [-0.7071, 0, 0.7071,0], $\lambda_{0}=-293 ;[0,0.7071,0,-0.7071], \lambda_{1}=-293 ;[0,0.7071,0,0.7071], \lambda_{2}=293$; $[-0.7071,0,-0.7071,0], \lambda_{3}=293$;

- the note la $\left(\mathrm{A}^{1}\right)$ with its frequency $440 \mathrm{~Hz}$ is represented by the square matrix $440 \mathrm{e}_{3}$ simultaneously with its 4 eigenvectors and their eigenvalues $\lambda_{\mathrm{i}}$ : $[0,-0.7071,0.7071$, $0], \lambda_{0}=-440 ;[0.7071,0,0,-0.7071], \lambda_{1}=-440 ;[0.7071,0,0,0.7071], \lambda_{2}=440$; $[0,0.7071,0.7071,0], \lambda_{3}=440$.

One can see that in such presentation each note of the considered musical scale has its own orthogonal system of eigenvectors and eigenvalues in multi-dimensional configurational space of this scale. Accordingly, the sequence of sound members of this musical fragment can be considered as a sequence of transformations of the orthogonal system of eigenvectors and eigenvalues of a previous note into an orthogonal system of eigenvectors and eigenvalues of a subsequent note. The transition of one such system of eigenvectors to another system is carried out using orthogonal matrices representing proper or improper rotations in the considered multi-dimensional space.

In the proposed algebraic approach, each chord can be represented by the sum of $2^{n}$-dimensional hyperbolic numbers representing its notes. For example, in the considered tetratonic scale, a chord of notes with sound frequencies 130,196 and $440 \mathrm{~Hz}$ can be represented by the aggregated hyperbolic number $130 \mathrm{e}_{0}+196 \mathrm{e}_{1}+440 \mathrm{e}_{3}$, which has the following 4 eigenvectors and eigenvalues: $[0.5,-0.5,0.5,-0.5], \lambda_{0}=-506 ;[-0.5,-0.5,0.5,0.5]$, $\lambda_{1}=-114 ; \quad[0.5,-0.5,-0.5,0.5], \lambda_{2}=374 ;[-0.5,-0.5,-0.5,-0.5], \lambda_{3}=766$. The described approach allows algebraic studying musical harmony and it can be applied for cases of 
22

various musical scales (including "genetic scales" described in [Petoukhov, 2008; Petoukhov, He, 2010]).

Let us additionally explain a specificity of this approach by an example of its usage for modeling a short sequence of separate notes and musical chords in Fig. 8.1. We will assume that its note frequencies correspond to the case of Pythagorean 7-stages scale in Fig. 7.1.

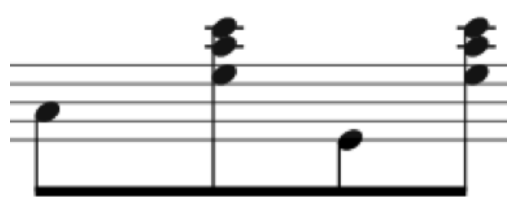

Fig. 8.1. An arbitrary example of a sequence of separate notes and musical chords.

Inside the first octave, which begins from $260 \mathrm{~Hz}$, the set of 7 notes of Pythagorean scale from Fig. 7.1 has sound frequencies shown in Fig. 8.2.

\begin{tabular}{|l|l|l|l|l|l|l|}
\hline do $\left(\mathrm{C}^{1}\right)$ & re $\left(\mathrm{D}^{1}\right)$ & mi $\left(\mathrm{E}^{1}\right)$ & fa $\left(\mathrm{F}^{1}\right)$ & sol $\left(\mathrm{G}^{1}\right)$ & la $\left(\mathrm{A}^{1}\right)$ & si $\left(\mathrm{B}^{1}\right)$ \\
\hline 260 & 293 & 330 & 348 & 392 & 440 & 495 \\
\hline
\end{tabular}

Fig. 8.2. Sound frequencies in $\mathrm{Hz}$ inside the first octave for 7 notes of the Pythagorean musical scale from Fig. 7.1.

In the considered approach, each of notes of this scale is connected with one of basis units of 8-dimensional hyperbolic number $a_{0} j_{0}+a_{1} j_{1}+a_{2} j_{2}+a_{3} j_{3}+a_{4} j_{4}+a_{5} j_{5}+a_{6} j_{6}+a_{6} j_{6}+a_{7} j_{7}$ for example in the following way:

- The coefficient a corresponds to the sound frequency of the note "do (C)" in corresponding octave $\left(\mathrm{a}_{0}=260 \mathrm{~Hz}\right.$ for the case "do $\left(\mathrm{C}^{1}\right)$ "). This note is expressed as 8 -dimensional hyperbolic number $\mathrm{a}_{0} \mathrm{j}_{0}$ with its matrix representation in a form of a sparce $(8 * 8)$-matrix;

- The coefficient $a_{1}$ corresponds to the sound frequency of the note "re (D)" in a corresponding octave $\left(a_{1}=293 \mathrm{~Hz}\right.$ for the case "re $\left(D^{1}\right)$ "). This note is expressed as 8-dimensional hyperbolic number $\mathrm{a}_{1} \mathrm{j}_{1}$;

- The coefficient a corresponds to the sound frequency of the note "mi (E)" in a corresponding octave $\left(\mathrm{a}_{2}=330 \mathrm{~Hz}\right.$ for the case " $\mathrm{mi}\left(\mathrm{E}^{1}\right)$ "). This note is expressed as 8 -dimensional hyperbolic number $\mathrm{a}_{2} \mathrm{j}_{2}$;

- The coefficient $a_{3}$ corresponds to the sound frequency of the note "fa (F)" in a corresponding octave $\left(\mathrm{a}_{3}=348 \mathrm{~Hz}\right.$ for the case " $\mathrm{fa}\left(\mathrm{F}^{1}\right)$ "). This note is expressed as 8-dimensional hyperbolic number $\mathrm{a}_{3} \mathrm{j}_{3}$;

- The coefficient a corresponds to the sound frequency of the note "sol (G)" in a corresponding octave $\left(\mathrm{a}_{4}=392 \mathrm{~Hz}\right.$ for the case "sol $\left(\mathrm{G}^{1}\right)$ "). This note is expressed as 8-dimensional hyperbolic number $\mathrm{a}_{4} \mathrm{j}_{4}$;

- The coefficient as corresponds to the sound frequency of the note "la (A)" in a corresponding octave $\left(a_{5}=440 \mathrm{~Hz}\right.$ for the case "la $\left(\mathrm{A}^{1}\right)$ "). This note is expressed as 8-dimensional hyperbolic number asj5; 
- The coefficient $\mathrm{a}_{6}$ corresponds to the sound frequency of the note "si (B)" in a corresponding octave $\left(\mathrm{a}_{6}=495 \mathrm{~Hz}\right.$ for the case "si $\left(\mathrm{B}^{1}\right)$ "). This note is expressed as 8-dimensional hyperbolic number $\mathrm{a}_{6} \mathrm{j} 6$;

- The coefficient $\mathrm{a}_{7}=0$ corresponds to the case of silence.

All these representations of separate notes of the musical scale have also their matrix represenations in a form of sparce ( $8 * 8$ )-matrices (Fig. 8.3) with their eigenvectors and eigenvalues.

\begin{tabular}{|c|c|c|c|}
\hline $\mathrm{a}_{0} 00000000$ & $\begin{array}{llllllll}0 & \mathrm{a}_{1} & 0 & 0 & 0 & 0 & 0 & 0\end{array}$ & $\begin{array}{lllllllll}0 & 0 & \mathrm{a}_{2} & 0 & 0 & 0 & 0 & 0\end{array}$ & $\begin{array}{llllllll}0 & 0 & 0 & \mathrm{a}_{3} & 0 & 0 & 0 & 0\end{array}$ \\
\hline $0 \mathrm{a}_{0} 0000000$ & $\mathrm{a}_{1} \begin{array}{lllllll}0 & 0 & 0 & 0 & 0 & 0 & 0\end{array}$ & $\begin{array}{lllllllll}0 & 0 & 0 & \mathrm{a}_{2} & 0 & 0 & 0 & 0\end{array}$ & 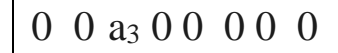 \\
\hline $00 \mathrm{a}_{0} 00000$ & $\begin{array}{llllllll}0 & 0 & 0 & a_{1} & 0 & 0 & 0 & 0\end{array}$ & $\mathrm{a}_{2} \begin{array}{llllllll}0 & 0 & 0 & 0 & 0 & 0 & 0\end{array}$ & $\begin{array}{llllllll}0 & \mathrm{a}_{3} & 0 & 0 & 0 & 0 & 0 & 0\end{array}$ \\
\hline $000 \mathrm{a}_{0} 0000$ & $\begin{array}{llllllll}0 & 0 & \mathrm{a}_{1} & 0 & 0 & 0 & 0 & 0\end{array}$ & $\begin{array}{llllllll}0 & \mathrm{a}_{2} & 0 & 0 & 0 & 0 & 0 & 0\end{array}$ & $\mathrm{a}_{3} \begin{array}{llllllll}0 & 0 & 0 & 0 & 0 & 0 & 0\end{array}$ \\
\hline $0000 \mathrm{a}_{0} 000$ & $\begin{array}{lllllllllllllll}0 & 0 & 0 & 0 & 0 & a_{1} & 0 & 0\end{array}$ & 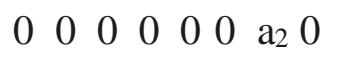 & $\begin{array}{llllllll}0 & 0 & 0 & 0 & 0 & 0 & 0 & a_{3}\end{array}$ \\
\hline $00000 \mathrm{a}_{0} 00$ & $\begin{array}{lllllllll}0 & 0 & 0 & 0 & \mathrm{a}_{1} & 0 & 0 & 0\end{array}$ & $\begin{array}{llllllll}0 & 0 & 0 & 0 & 0 & 0 & 0 & a_{2}\end{array}$ & $\begin{array}{llllllll}0 & 0 & 0 & 0 & 0 & 0 & a_{3} & 0\end{array}$ \\
\hline $000000 \mathrm{a}_{0} 0$ & $\begin{array}{llllllll}0 & 0 & 0 & 0 & 0 & 0 & 0 & a_{1}\end{array}$ & $\begin{array}{lllllllll}0 & 0 & 0 & 0 & a_{2} & 0 & 0 & 0\end{array}$ & $\begin{array}{llllllllllllllll}0 & 0 & 0 & 0 & 0 & \mathrm{a}_{3} & 0 & 0\end{array}$ \\
\hline $0000000 \mathrm{a}_{0}$ & $\begin{array}{lllllll}0 & 0 & 0 & 0 & 0 & 0 & a_{1}\end{array}$ & $\begin{array}{lllllllll}0 & 0 & 0 & 0 & 0 & \mathrm{a}_{2} & 0 & 0\end{array}$ & $\begin{array}{llllllll}0 & 0 & 0 & 0 & a_{3} & 0 & 0 & 0\end{array}$ \\
\hline $\begin{array}{llllllllllllllll}0 & 0 & 0 & 0 & \mathrm{a}_{4} & 0 & 0 & 0\end{array}$ & $\begin{array}{llllllll}0 & 0 & 0 & 0 & 0 & \mathrm{a}_{5} & 0 & 0\end{array}$ & $\begin{array}{llllllll}0 & 0 & 0 & 0 & 0 & 0 & \mathrm{a}_{6} & 0\end{array}$ & $\begin{array}{llllllll}0 & 0 & 0 & 0 & 0 & 0 & 0 & a_{7}\end{array}$ \\
\hline $\begin{array}{lllllllll}0 & 0 & 0 & 0 & 0 & a_{4} & 0 & 0\end{array}$ & $\begin{array}{llllllll}0 & 0 & 0 & 0 & \text { a } 5 & 0 & 0 & 0\end{array}$ & $\begin{array}{llllllll}0 & 0 & 0 & 0 & 0 & 0 & 0 & a_{6}\end{array}$ & 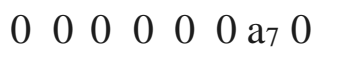 \\
\hline $\begin{array}{llllllll}0 & 0 & 0 & 0 & 0 & 0 & a_{4} & 0\end{array}$ & $\begin{array}{llllllll}0 & 0 & 0 & 0 & 0 & 0 & 0 & a_{5}\end{array}$ & $\begin{array}{llllllllll}0 & 0 & 0 & 0 & \mathrm{a}_{6} & 0 & 0 & 0\end{array}$ & $\begin{array}{llllllll}0 & 0 & 0 & 0 & 0 & a_{7} & 0 & 0\end{array}$ \\
\hline $\begin{array}{llllllll}0 & 0 & 0 & 0 & 0 & 0 & 0 & a_{4}\end{array}$ & 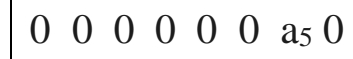 & $\begin{array}{llllllll}0 & 0 & 0 & 0 & 0 & \mathrm{a}_{6} & 0 & 0\end{array}$ & $\begin{array}{llllllllll}0 & 0 & 0 & 0 & \text { a } & 0 & 0 & 0\end{array}$ \\
\hline $\mathrm{a}_{4} \begin{array}{lllllll}0 & 0 & 0 & 0 & 0 & 0 & 0\end{array}$ & 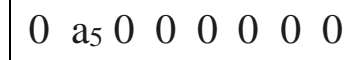 & $\begin{array}{llllllll}0 & 0 & \mathrm{a}_{6} & 0 & 0 & 0 & 0 & 0\end{array}$ & $\begin{array}{llllllll}0 & 0 & 0 & a_{7} & 0 & 0 & 0 & 0\end{array}$ \\
\hline $\begin{array}{llllllll}0 & \mathrm{a}_{4} & 0 & 0 & 0 & 0 & 0 & 0\end{array}$ & a $\begin{array}{lllllll}0 & 0 & 0 & 0 & 0 & 0 & 0\end{array}$ & $\begin{array}{llllllll}0 & 0 & 0 & \mathrm{a}_{6} & 0 & 0 & 0 & 0\end{array}$ & 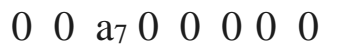 \\
\hline $\begin{array}{llllllll}0 & 0 & \mathrm{a}_{4} & 0 & 0 & 0 & 0 & 0\end{array}$ & 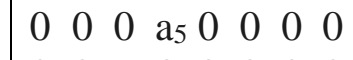 & $\mathrm{a}_{6} \begin{array}{lllllll}0 & 0 & 0 & 0 & 0 & 0 & 0\end{array}$ & 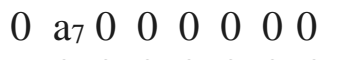 \\
\hline $\begin{array}{llllllll}0 & 0 & 0 & \mathrm{a}_{4} & 0 & 0 & 0 & 0\end{array}$ & $\begin{array}{llllllll}0 & 0 & \mathrm{a}_{5} & 0 & 0 & 0 & 0 & 0\end{array}$ & $\begin{array}{llllllll}0 & \mathrm{a}_{6} & 0 & 0 & 0 & 0 & 0 & 0\end{array}$ & $\mathrm{a}_{7} \begin{array}{lllllll}0 & 0 & 0 & 0 & 0 & 0 & 0\end{array}$ \\
\hline
\end{tabular}

Fig. 8.3. Matrices $a_{0} j_{0}, a_{1} j_{1} a_{2} j_{2}, a_{3} j_{3}, a_{4} j_{4}, a_{5} j_{5}, a_{6} j_{6}, a_{6} j_{6}$ and $a_{7} j_{7}$, which represent separate notes of the musical scale from Fig. 8.2 as components of 8-dimensional hyperbolic number.

Returning to the musical fragment in Fig. 8.1, one can see that its first note "la $\left(\mathrm{A}^{1}\right)$ " and its third note "mi $\left(\mathrm{E}^{1}\right)$ " are now represented as $440 \mathrm{j}_{5}$ and $330 \mathrm{j}_{3}$ correspondingly. The chord of this fragment is represented by the sum of the three notes " $m i\left(E^{2}\right)$ ", "la $\left(A^{2}\right)$ " and "do $\left(\mathrm{C}^{3}\right)$ " included in its composition that is by the 8-dimensional hyperbolic number $660 \mathrm{j}_{3}+880 \mathrm{j}_{5}+1040 \mathrm{j}_{0}$. From this standpoint this fragment is the sequence (8.1) of $(8 * 8)$-matrix representations of 8 -dimensional hyperbolic numbers:

$$
\left(440 \mathrm{j}_{5}\right) \rightarrow\left(660 \mathrm{j}_{3}+880 \mathrm{j}_{5}+1040 \mathrm{j}_{0}\right) \rightarrow\left(330 \mathrm{j}_{3}\right) \rightarrow\left(660 \mathrm{j}_{3}+880 \mathrm{j}_{5}+1040 \mathrm{j}_{0}\right)
$$

Each (8*8)-matrix member of this sequence has an orthogonal set of its 8 eigenvectors with appropriate eigenvalues. For example, the first member $\left(440 \mathrm{j}_{5}\right)$ has the following 8 eigenvectors:

- $[0,-0.7071,0,0,0.7071,0,0,0]$ with eigenvalues: $-440,0,0,0,0,0,0,0$;

- $[0,0,0,-0.7071,0,0,0.7071,0]$ with eigenvalues: $0,-440,0,0,0,0,0,0$;

- $[0.7071,0,0,0,0,-0.7071,0,0]$ with eigenvalues: $0,0,-440,0,0,0,0,0$; 
24

- $[0,0,0.7071,0,0,0,0,-0.7071]$ with eigenvalues: $0,0,0,-440,0,0,0,0$;

- $[0.7071,0,0,0,0,0.7071,0,0]$ with eigenvalues: $0,0,0,0,440,0,0,0$;

- $[0,0,0,0.7071,0,0,0.7071,0]$ with eigenvalues: $0,0,0,0,0,440,0,0$;

- $[0,0,0.7071,0,0,0,0,0.7071]$ with eigenvalues: $0,0,0,0,0,0,440,0$;

- $[0,0.7071,0,0,0.7071,0,0,0]$ with eigenvalues: $0,0,0,0,0,0,0,440$.

The eigenvectors of the second member and the fourth member in (8.1) that is the $(8 * 8)$-matrix $\left(660 \mathrm{j}_{3}+880 \mathrm{j}_{5}+1040 \mathrm{j}_{0}\right)$ has quite another orthogonal set of its 8 eigenvectors:

- $[0,-0.5,0.5,0,0.5,0,0,-0.5]$ with eigenvalues: $1040,0,0,880,0,880,0,0$;

- $[-0.5,0,0,0.5,0,0.5,-0.5,0]$ with eigenvalues: $0,1040,880,0,880,0,0,0$;

- [-0.6946, -0.1323, 0, 0, 0, 0, 0.6946, 0.1323] with eigenvalues: $0,880,1040,0,0,0,0,880$;

- $[0,0,0.7071,0,-0.7071,0,0,0]$ with eigenvalues: $880,0,0,1040,0,0,880,0$;

- $[0,0,0,-0.7071,0,0.7071,0,0]$ with eigenvalues: $0,880,0,0,1040,0,0,880$;

- $[-0.1323,0.6946,0,0,0,0,0.1323,-0.6946]$ with eigenvalues: $880,0,0,0,0,1040,880,0$;

- [0.0083, 0.4999, 0.4999, 0.0083, 0.4999, 0.0083, 0.0083, 0.4999] with eigenvalues: $0,0,0$, 880, 0, 880, 1040, 0;

- $[0.4999,-0.0083,-0.0083,0.4999,-0.0083,0.4999,0.4999,-0.0083]$ with eigenvalues: 0,0 , $880,0,880,0,0,1040$.

The eigenvectors of the third member in (8.1), that is the $(8 * 8)$-matrix $330 \mathrm{j} 3$, has its own orthogonal set of 8 eigenvectors:

- $[0,-0.7071,0.7071,0,0,0,0,0]$ with eigenvalues: $-330,0,0,0,0,00,0$;

- [0.7071, 0, 0, -0.7071, 0, 0, 0, 0] with eigenvalues: $0,-330,0,0,0,0,0,0$;

- $[0,0,0,0,0,-0.7071,0.7071,0]$ with eigenvalues: $0,0,-330,0,0,0,0,0$;

- $[0,0,0,0,0.7071,0,0,-0.7071]$ with eigenvalues: $0,0,0,-330,0,0,0,0$;

- $[0,0,0,0,0,0.7071,0.7071,0]$ with eigenvalues: $0,0,0,0,330,0,0,0$;

- $[0.7071,0,0,0.7071,0,0,0,0]$ with eigenvalues: $0,0,0,0,0,330,0,0$;

- $[0,0,0,0,0.7071,0,0,0.7071]$ with eigenvalues: $0,0,0,0,0,0,330,0$;

- $[0,0.7071,0.7071,0,0,0,0,0]$ with eigenvalues: $0,0,0,0,0,0,0,330$.

But musical works are based not only on the sound frequencies of notes of a particular musical system but also on a system of note durations: 1, 1/2, 1/4, 1/8, 1/16, 1/32, 1/64, 1/128 (with their traditional names: a whole note, a half note, a quarter note, an eighth note, a sixthteenth note, ...). In musical pieces each note is a symbiosis of a sound frequency and one of these durations. Correspondingly in the proposed approach on the foundation of $2^{n}$-dimensional hyperbolic numbers, such symbiosis can be expressed as a sum of approproate basis units of these numbers. Let us explain this.

One can take 2-dimensional hyperbolic number $\mathrm{d}_{0}+1 / 2 * \mathrm{~d}_{1}$ in its matrix representation $[1,1 / 2 ; 1 / 2,1]$. Rising this matrix into appropriate tensor power $n$ automatically gives matrix representation of $2^{n}$-dimensional hyperbolic number with coefficients of its basis units $1,1 / 2$, $1 / 4,1 / 8,1 / 16,1 / 32,1 / 64,1 / 128$. In the simplest case, if one wants to have this set of durations as the set of coefficients of basis units, it is enough to take 8-dimensional hyperbolic number $\mathrm{d}_{0}+1 / 2 * \mathrm{~d}_{1}+1 / 4 * \mathrm{~d}_{2}+1 / 8 * \mathrm{~d}_{3}+1 / 16 * \mathrm{~d}_{4}+1 / 32 * \mathrm{~d}_{5}+1 / 64 * \mathrm{~d}_{6}+1 / 128 * \mathrm{~d}_{7}$.

To construct an algebraic symbiosis of note sound frequencies of any musical scale (Pythagorean, equal tempered scale, etc) and of the standard set of note durations 1, 1/2, 1/4, $1 / 8,1 / 16,1 / 32,1 / 64,1 / 128$, one can take enough long $2^{n}$-dimensional hyperbolic number, whose first part contains basis units with coefficients identical to sound frequencies of the 
musical scale and whose second part contains basis units with coefficients identical to these durations. For example, in the case of Pythagorean musical scale (Fig. 8.2), which contains 7 note frequencies, it is enough to take 16-dimensional hyperbolic number $S_{16}$ (8.2), whose first part contains basis units with coefficients identical to note frequencies and the second part contains basis units with coefficients identical to note durations:

$$
\begin{gathered}
\mathrm{S}_{16}=260 * \mathrm{~s}_{0}+293 * \mathrm{~s}_{1}+330 * \mathrm{~s}_{2}+348 * \mathrm{~s}_{3}+392 * \mathrm{~s}_{4}+440 * \mathrm{~s}_{5}+495 * \mathrm{~s}_{6}+0 * \mathrm{~s}_{7}+ \\
1 * \mathrm{~s}_{8}+1 / 2 * \mathrm{~s}_{9}+1 / 4 * \mathrm{~s}_{10}+1 / 8 * \mathrm{~s}_{11}+1 / 16 * \mathrm{~s}_{12}+1 / 32 * \mathrm{~s}_{13}+1 / 64 * \mathrm{~s}_{14}+1 / 128 * \mathrm{~s}_{15}
\end{gathered}
$$

In (8.2) the member $0 * \mathrm{~s}_{7}$ represents silence. Correspondingly a symbiosis of separate sound frequency and separate note duration has a common orthogonal system of eigenvectors and eigenvalues. For example, the symbiosis of the note do $\left(\mathrm{C}^{1}\right)$ and the duration $1 / 4$ is expresses by the sum $260 * s_{0}+1 / 4 * s_{10}$, whose matrix representation has a common orthogonal system of 16 eigenvalues.

In the case of equal tempered scale, which has 12 note sound frequencies, the octave is devided into 12 parts with a ratio equal to the 12 th root of 2 . In the proposed approach, to consider a symbiosis of this frequencies scale and durations, we need at least 32-dimensional hyperbolic number (8.3). Its first part contains basis units with coefficients iden-tical to note frequencies (expressed in hertz or in ratios related with the 12th root of 2) and its second part contains basis units with coefficients identical to note durations. Many basis units in (8.3) have zero coefficients since it is enough 20 members of this 32-dimensional number to express the symbiosis of the set of 12 sound frequencies and the set of 8 durations.

$$
\begin{aligned}
\mathrm{s}_{32} & =1 * \mathrm{~s}_{0}+2^{1 / 12 *} \mathrm{~s}_{1}+2^{2 / 12 *} \mathrm{~s}_{2}+2^{3 / 12 *} \mathrm{~s}_{3}+2^{4 / 12 *} \mathrm{~s}_{4}+2^{5 / 12 *} \mathrm{~s}_{5}+2^{6 / 12 *} \mathrm{~s}_{6}+2^{7 / 12 *} \mathrm{~s}_{7} \\
& +2^{8 / 12 *} \boldsymbol{s}_{8}+2^{9 / 12 *} \mathrm{~s}_{9}+2^{10 / 12 *} \mathrm{~s}_{10}+2^{11 / 12 *} \mathrm{~s}_{11}+0 * \mathrm{~s}_{12}+0 * \mathrm{~s}_{13}+0 * \mathrm{~s}_{14}+0 * \mathrm{~s}_{15}+ \\
& 1 * \mathrm{~s}_{16}+1 / 2 * \mathrm{~s}_{17}+1 / 4 * \mathrm{~s}_{18}+1 / 8 * \mathrm{~s}_{19}+1 / 16 * \mathrm{~s}_{20}+1 / 32 * \mathrm{~s}_{21}+1 / 64 * \mathrm{~s}_{22}+1 / 128 * \mathrm{~s}_{23} \\
& +0 * \mathrm{~s}_{24}+0 * \mathrm{~s}_{25}+0 * \mathrm{~s}_{26}+0 * \mathrm{~s}_{27}+0 * \mathrm{~s}_{28}+0 * \mathrm{~s}_{29}+0 * \mathrm{~s}_{2} \mathrm{~s}_{31}
\end{aligned}
$$

One can remind that each kind of $2^{n}$-dimensional hyperbolic numbers under fixed $n$ has its own hyper-alphabet of $2^{n}$ orthogonal systems of its $2^{n}$ basis units. Transitions from one member of such hyper-alphabet to other members are determined by transformations of mentioned orthogonal rotations (see above Section 6). From the proposed algebraic point of view, any musical piece is related with a sequence of the mentioned rotations of orthogonal systems of eigenvectors inside appropriate multi-dimensional vector space. These rotations of elements of music generate some associations with rotations (or whirling, or spin) of dancing pairs under dance music. Perhaps classical rotating movements of dancing pairs are related in some degree with mentioned rotations of members of algebraic hyper-alphabets of music. One can add that all movements of separate parts of our body skelet are provided by their rotations in joints.

It seems important to note the following. The traditional use of one-dimensional numbers in musicology made it possible to study the relationship of musical sound frequencies in musical systems and chords in line with the Pythagorean theory of the relationship of musical frequencies with dividing strings into parts. But it is obvious that knowledge of only the harmonious interrelations of sound frequencies is not enough to reveal the harmony of the musical work as a whole. For example, you can take two pieces of music, which use the same set of sound frequencies, but - because of different sequences of the same musical sounds in 
26

them - one piece will provides a charming effect on the listener, and the other will leave him indifferent or will cause a negative emotion. This indicates that in musical works there is some other - additional - type of harmony, reflected in the transitions between sounds and providing harmonical development of the theme of a musical work (musical plasticity).

The author's proposal of an application of $2^{n}$-dimensional hyperbolic numbers with their matrix forms of representation, which fully preserves the Pythagorean ideas about the harmonical relationship of musical frequencies, allows studying a completely different type of music harmony: harmony of transitions in a sounds sequence of a musical work, that is, harmony of the development of the theme of a musical work (musical plasticity). From the proposed algebraic point of view, a musical piece can be considered as a sequence of orthogonal transformations of the bases of multidimensional spaces. This new study is possible due to introducing quite new personages into mathematical musicology: matrix presentations of $2^{n}$-dimensional hyperbolic numbers with orthogonal systems of eigenvectors of their basis units to study such plasticity.

In the proposed approach, music appears as a game with $2^{n}$-dimensional hyperbolic numbers. It reminds some ideas of the book "The Glass Bead Game" of Nobel laureate in literature by Hermann Hesse, where deep thoughts on interrelations of mathematics and music are presented [Hesse, 2002].

Similar algebraic approaches are possible for analyzing DNA sequences, but corresponding author's results will be published some later.

\section{Advantages of matrix representations of hyperbolic numbers}

The matrix forms of presentation of $2^{n}$-dimensional hyperbolic numbers deserve a special attention since they have the following useful properties:

1. This presentation form is based on symmetric matrices, which are closely related with the theory of resonances of oscillatory systems, having many degrees of freedom, and also with Punnett squares from Mendelian genetics of inheritance of traits in living organisms [Petoukhov, 2011, 2015, 2016]. Symmetrical matrices are related with the theory of resonance of L. Pauling whose book [Pauling, 1940] about this theory in structural chemistry is the most quoted among scientific books of the XX century. The actual molecule, as Pauling proposed, is a sort of hybrid, a structure that resonates between the two alternative extremes; and whenever there is a resonance between the two forms, the structure is stabilized. Pauling claimed that living organisms are chemical in nature, and resonances in their molecules should be very essential for biological phenomena. In general, quantum mechanics was emerged and developed largely as a science about resonances in microworld. Thus, the concept of systemresonance genetics (or spectral-resonance genetics) creates models of genetic phenomena on the same language of frequencies and resonances, on which models in quantum mechanics are based. In addition to this, it uses the same matrix language, on which "matrix mechanics" of Werner Heisenberg has been created; it is historically the first form of quantum mechanics, which retains its value to this day.

2. These symmetric matrices are Hermitian (self-adjoint) matrices, which play an important role in quantum mechanics. By this reason they can be used in development of applications of ideas and methods of quantum mechanics and quantum informatics in the field of bioinformatics and algebraic biology. In this connection some of 
author's works [Petoukhov, 2018a,b, 2019a,b; Petoukhov, Petukhova, Svirin, 2019] are devoted to using formalisms of quantum mechanics and quantum informatics in bioinformatics and algebraic biology including analysis of long genetic and and literary texts. For example, in long DNA sequences of nucleobases, where complementary nucleobases $\mathrm{C}$ and $\mathrm{G}$ (A and T) are linked by 3 (2) hydrogen bonds, $2^{n}$-dimensional hyperbolic numbers $[\% 3, \% 2 ; \% 2, \% 3]^{(n)}$ (where $\% 3$ and $\% 2$ denote percentages of numbers 3 and 2 of hydrogen bonds in the analyzed DNA sequence; $n=2,3,4,5)$ effectively models percentages of monoplets, doublets, triplets, tetraplets and pentaplets of these numbers 3 and 2 of hydrogen bonds [Petoukhov, 2018].

3. These symmetric matrices can be interpreted as metric tensors, which are main invariants in Riemanian geometry and which can be used in the theory of morpho-resonance morphogenesis [Petoukhov, 2008, 2015, 2016];

4. These symmetric matrices are related with hyperbolic rotations [ $\operatorname{sh} \mathrm{x}, \mathrm{ch} \mathrm{x} ; \mathrm{ch} \mathrm{x}, \mathrm{sh} \mathrm{x}$, which are particular cases of hyperbolic numbers and are connected with the theory of biological phyllotaxis laws [Bodnar, 1992, 1994; Stakhov, 2009], with problems of locomotion control [Smolyaninov, 2000], with the main psychophysical law of Weber-Fechner (see above and also in [Petoukhov, 2016]), with Lorenz transformations in the special theory of relativity;

5. These symmetric matrices are related with the theory of solitons of sine-Gordon equation [Petoukhov, 1999, 2008; Petoukhov, He, 2009]. Such solitons are the only relativistic type of solitons; they were put forward for the role of the fundamental type of solitons of living matter in the book [Petoukhov, 1999].

Symmetric matrices possess a wonderful property to express resonances [Bellman, 1960; Balonin, 2000]. The expression $y=A * S$ models the transmission of a signal $S$ via an acoustic system A, represented by a relevant matrix A. If an input signal is a resonant tone, then the output signal will repeat it with a precision up to a scale factor $y=\lambda * S$ by analogy with a situation when a musical string sounds in unison with the neighboring vibrating string. In the case of a matrix A, its number of resonant tones $S_{\mathrm{i}}$ corresponds to its size. They are termed its eigenvectors, and the scale factors $\lambda_{\mathrm{i}}$ with them are termed its eigenvalues or, briefly, spectrum A. One of the main tasks of the theory of oscillations is a determination of natural frequencies (mathematically, eigenvalues of operators) and the natural forms of oscillations of bodies. To find all the eigenvalues $\lambda_{\mathrm{i}}$ and eigenvectors of the matrix $\mathrm{A}$, which are defined by the matrix equation $\mathrm{A}^{*} \mathrm{~s}=\lambda^{*} \mathrm{~s}$, the "characteristic equation" of the matrix $\mathrm{A}$ is analyzed: $\operatorname{det}(\mathrm{A}-\mathrm{E})=0$, where $\mathrm{E}-$ the identity matrix (see more in [Petoukhov, 2016]). Matrices, which are relevant to the various problems of the theory of oscillations, are usually symmetric real matrices [Gladwell, 2004]. Such matrices have real eigenvalues and their eigenvectors are orthogonal.

Symmetric matrices representing hyperbolic numbers are simultaneously metric tensors by their structure. Metric tensors are main invariants of Riemanian geometry, which can be used for modelling inherited curvilinear forms of biological bodies. By definition, the metric tensor in the $n$-dimensional affine space with the scalar multiplication introduced is defined by the nondegenerate matrix $\left\|g_{i j}\right\|$ under the condition of symmetry $g_{i j}=g_{j i}$ [Rashevskij, 1964], which is satisfied by the structure of bisymmetric matrices of hyperbolic numbers. The coordinates $g_{i j}$ of the metric tensor are the pairwise scalar products of vectors of the frame, on which it is built. If we extract the square root from a bisymmetric matrix, we get a square matrix whose columns are vectors of this frame. It is interesting that the extraction of the 
28

square root from quint matrices of $2^{n}$-dimensional hyperbolic numbers $[3,2 ; 2,3]^{(n)}$, which has integer components, get square matrices of $2^{n}$-dimensional hyperbolic numbers $\left[\varphi, \varphi^{-1} ; \varphi^{-1}, \varphi\right]^{(n)}$ whose components are irrational numbers of the golden section $\varphi=\left(1+5^{0.5}\right) / 2=1,618 \ldots$ in integer powers; the golden section $\varphi$ is famous in the aesthetics of proportions and described by many authors in a series of inherited physiological systems [Petoukhov, 2008; Petoukhov, He, 2010]. It means that metric tensors, having forms of quint matrices of hyperbolic numbers, are built on a frame of "golden" vectors, all components of which are equal to the golden sections in integer powers.

\section{$102^{n}$-dimensional hyperbolic numbers and phenomenological rules of probabilities in genetics}

The author revealed that in some cases it is possible to use $2^{n}$-dimensional hyperbolic numbers and their matrix representations for modeling some phenomenological rules in biology, first of all, in genetics. In this cases the tensor family of symmetric matrices $[\% \mathrm{~S}, \% \mathrm{~W} ; \% \mathrm{~W}, \% \mathrm{~S}]^{(n)}$ is under consideration, where $\% \mathrm{~S}$ and $\% \mathrm{~W}$ refer to percentages of biological realisation of some events denoted by symbols $\mathrm{S}$ and $\mathrm{W}(\% \mathrm{~W}+\% \mathrm{~S}=100 \%)$.

This tensor family contains matrix representations of 2-dimensional hyperbolic numbers $\% \mathrm{~S}+\% \mathrm{~W}^{*} \mathrm{j}_{1} ;$ of 4-dimensional hyperbolic numbers $\% \mathrm{~S} * \% \mathrm{~S}+\% \mathrm{~S} * \% \mathrm{~W}^{*} \mathrm{j}_{1}+\% \mathrm{~W} * \% \mathrm{~S} * \mathrm{j}_{2}+$ $\% \mathrm{~W}^{*} \% \mathrm{~W}^{* \mathrm{j}_{3}}$; of 8 -dimensional hyperbolic numbers, etc. Expressions like as $\% \mathrm{~S} * \% \mathrm{~S}$, $\% \mathrm{~S} * \% \mathrm{~W}, \% \mathrm{~W} * \% \mathrm{~W}$ can be considered as percentages of realisation of doublets $\mathrm{SS}, \mathrm{SW}$, $\mathrm{WW}$ in chains of these events.

For example, the phenomenological rules described in [Petoukhov, 2011, 2018a,b, 2019b] can be modeled on the foundation of this approach. Details of such modeling will be published some later.

\section{Fractal-like multi-dimensional configurational spaces of hyperbolic types}

This Section is devoted to the use of $2^{n}$-dimensional hyperbolic numbers for modeling heritable fractal-like biostructures, which are developing step by step in ontogenesis of biological bodies.

Living bodies in a course of their ontogenesis from the embryonic state to the mature state gradually increase the number of body parts. Accordingly, the number of parameters, characterizing the developing body, increases. This leads to appropriate phased increasing a dimensionality of a configurational space of parameters of the body. In many cases of such ontogenetic development one can see the following iterative process: body structural elements, which exist at a previous stage of ontogenesis, produce - at the next step of ontogenesis - new elements with similar structures (Fig. 11.1). In the result, after some repetitions of this ontogenetic procedure, complex fractal-like structure of the multi-level body appears. A multidimensional configurational space of parameters of such body has a fractal-like system of its different subspaces having similar patterns of parametric states. One of many examples of such phased producing a fractal-like structure of multi-level body is ontogenetic producing new and new dichotomic branches in some plants (Fig. 11.1, left). 


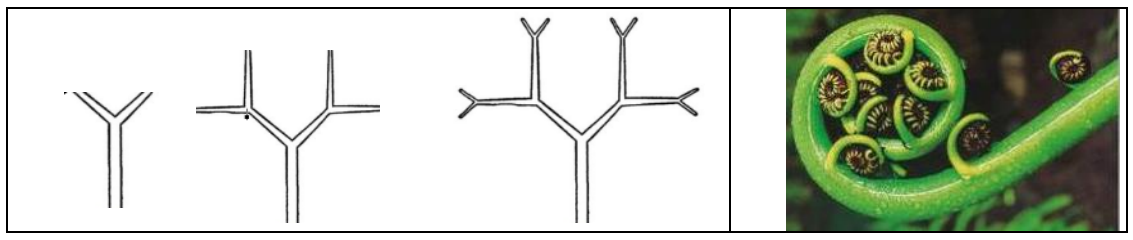

Fig. 11.1. Illustrations for the phased ontogenetic development of fractal-like biological structures (from https://studbooks.net/2365314/tehnika/istoriya poyavleniya razvitiya).

Regarding the theme of fractal-like structures in biological bodies, one can note a great number of publications is devoted to algorithmic creation of fractal-like geometric figures in spaces of a fixed (!) dimensionality, first of all, in 2-dimensional complex plane. There are also known works devoted to constructions of fractal geometric patterns on the plane of hyperbolic (or double) numbers [Pavlov, Panchelyuga, Panchelyuga, 2009a,b].

In contrast to these works, the author proposes an approach to model an algorithmic reproduction of patterns, which are similar each other, not in a space of a fixed dimensionality but in different subspaces of multidimensional configurational spaces of parameters of multi-level bodies under their phased ontogenetic development. Due to similarity of parametric structures in its different subspaces, each of considered configurational spaces becomes a fractal-like space in the whole.

The author notes the following possibility of modelling such multi-step ontogenetic development of biological objects and their configurational spaces, which receive new and new parameters and dimensionalities step by step. Let us take the matrix representation of hyperbolic number $\left[f_{1}(t), f_{2}(t) ; f_{2}(t), f_{1}(t)\right]$ whose components $f_{1}(t)$ and $f_{2}(t)$ are functions of time. Fig. 11.2 shows that if this $(2 * 2)$-matrix is tensor multiplied on the left by a hyperbolic number $[1,1 ; 1,1]$, which acts as a generator of additional dimensionalities of the configurational space, the result is $(4 * 4)$-matrix representing 4-dimensional hyperbolic number $\mathrm{f}_{1}(\mathrm{t}) * \mathrm{e}_{0}$ $+\mathrm{f}_{2}(\mathrm{t}) * \mathrm{e}_{1}+\mathrm{f}_{1}(\mathrm{t}) * \mathrm{e}_{2}+\mathrm{f}_{2}(\mathrm{t}) * \mathrm{e}_{3}$. This 4-dimensional configurational space repeats in its subspaces (namely the first plane on the basis vectors $\mathrm{e}_{0}$ and $\mathrm{e}_{1}$, and the second plane on the basis vectors $\mathrm{e}_{2}$ and $\mathrm{e}_{3}$ ) the same functions $\mathrm{f}_{1}(\mathrm{t})$ and $\mathrm{f}_{2}(\mathrm{t})$, which were in the initial 2-dimensional space.

$$
\left|\begin{array}{l}
11 \\
11
\end{array}\right| \otimes\left|\begin{array}{l}
f_{1}(t), f_{2}(t) \\
f_{2}(t), f_{1}(t)
\end{array}\right|=\left|\begin{array}{l}
f_{1}(t), f_{2}(t), f_{1}(t), f_{2}(t) \\
f_{2}(t), f_{1}(t), f_{2}(t), f_{1}(t) \\
f_{1}(t), f_{2}(t), f_{1}(t), f_{2}(t) \\
f_{2}(t), f_{1}(t), f_{2}(t), f_{1}(t)
\end{array}\right|=f_{1}(t) * e_{0}+f_{2}(t) * e_{1}+f_{1}(t) * e_{2}+f_{2}(t) * e_{3}
$$

Fig. 11.2. An initial step of a generation of a fractal-like $2^{n}$-dimensional space whose subspaces have identical contents. Here $\mathrm{e}_{0}, \mathrm{e}_{1}, \mathrm{e}_{2}$ and $\mathrm{e}_{3}$ are basis units from Fig. 2.5.

Repeating the required number of times this operation of the tensor multiplication on the left using the generator $[1,1 ; 1,1]$, we obtain a hierarchical tree of $2^{n}$-dimensional hyperbolic numbers and their corresponding $2^{n}$-dimensional configurational spaces for algorithmic modelling multi-step onthogenesis of a fractal-like morphogenetic construction. Different levels of this tree have subspaces with the same functions $f_{1}(t)$ and $f_{2}(t)$, which were 
30

in the initial 2-dimensional space; in this sense one can speech about a fractal-like structure of this hierarchy of multi-dimensional configurational spaces of parameters.

We briefly note that the noted generator $[1,1 ; 1,1]$ (Fig. 11.2) can be used in a more complicated form if its components are some functions of time $g_{i}(t)$, for example $\left[g_{1}(t), g_{2}(t)\right.$; $\mathrm{g}_{2}(\mathrm{t}), \mathrm{g}_{1}(\mathrm{t})$ ]. For modeling biological cyclic processes based on such fractal-like sets of subspaces, the case, in which the functions $\mathrm{f}_{1}(\mathrm{t}), \mathrm{f}_{2}(\mathrm{t}), \mathrm{g}_{1}(\mathrm{t})$ and $\mathrm{g}_{2}(\mathrm{t})$ are cyclic functions of time, is especially interesting.

\section{Pythagoras and the importance of the concept of number}

The notion of "number" is the main notion of mathematics and mathematical natural sciences. Pythagoras has formulated the famous idea: "Numbers rule the world" or "All things are numbers". This Pythagorean slogan arised not because that the number can express a quantity of objects. Pythagoras was engaged in figured numbers associated with geometric figures: triangular, square, 5-angled, 12-angled, etc. Seeing that different numbers can dictate different geometric shapes, he came up with the idea that numbers have an internal structure and able to organize the outside world according to their properties. In view of this idea, natural phenomena should be explained by means of systems of numbers; the systems of numbers play a role of the beginning for uniting all things and for expressing the harmony of nature [Kline, 1980]. For the Pythagoreans, the number expressed the "essence" of everything, and therefore the phenomena should be explained only with the help of numbers; it was numerical relations that served as the unifying principle of all things and expressed the harmony and order of nature.

Many prominent scientists and thinkers were supporters of this Pythagorean standpoint or of one similar to it. As W. Heisenberg noted, modern physics, where matrices are used as a higher form of numbers, is moving along the same path along which the Pythagoreans walked [Heisenberg, 1958]. Not without reason B. Russell noted that he did not know any other person who could exert such influence on the thinking of people as Pythagoras [Russell, 1945]. Taking this into account, one can believe that there is no more fundamental scientific idea in the world than this idea about a basic meaning of numbers.

Our research results and the proposed approach can be considered as a further development of this fundamental idea of Pythagoras in connection with the structural organization of the genetic system and inherited biological phenomena.

\section{Some concluding remarks}

The development of modern mathematical natural sciences is based on the use of certain mathematical tools. Mathematical tools of theoretical research can be compared with glasses for a visually impaired person: adequate glasses provide a person with a clear and beautiful picture of reality, which he had previously seen as blurred and hidden by fog. Darwin once wrote: "I have deeply regretted that I did not proceed far enough at least to understand something of the great leading principles of mathematics; for men thus endowed seem to have an extra sense" (this quotation is taken from [May, 2004]). 
This article attracts attention of researches to an important role of hyperbolic numbers and their matrix representations in algebraic modelling structural features of genetic phenomena. The author puts forward the hypothesis that hyper-alphabets of eigenvectors of matrix representations of basis units of $2^{n}$-dimensional hyperbolic numbers play a key role in transmitting biological information and that they can be considered as a foundation of coding information at different levels of biological organization. He believes that corresponding languages using such hyper-alphabets define many inherited phenomenological structures in biology including molecular genetic structures. In particular, using these hyper-alphabets gives new algebraic tools to study phenomenological genetic rules and also harmony of musical pieces. The proposed algebraic approach is connected with the theme of a grammar of biology mentioned above.

In the author's opinion, the proposed kind of mathematics is beautiful and it can be used for further developing of algebraic biology and informatics in accordance with the famous statement by P. Dirac, who taught that a creation of a physical theory must begin with the beautiful mathematical theory: "If this theory is really beautiful, then it necessarily will appear as a fine model of important physical phenomena. It is necessary to search for these phenomena to develop applications of the beautiful mathematical theory and to interpret them as predictions of new laws of physics" (this quotation is taken from [Arnold, 2007]). According to Dirac, all new physics, including relativistic and quantum, are developing in this way. One can suppose that this statement is also true for mathematical biology.

\section{Appendix I. Dyadic groups of binary numbers, modulo-2 addition and matrices of dyadic shifts}

This article has repeatedly used a special decomposition of bisymmetric $\left(2^{n} * 2^{n}\right)$-matrices, which represented them as a sum of $2^{n}$ sparse matrices, defining multiplication tables of corresponing algebras (Figs. 2.3, 2.4, 7.2, 8.3). Just these sparce matrices represented the basic units of hyperbolic numbers. This Appendix explains what this special kind of decomposition is.

Bisymmetric matrix representations of $2^{n}$-dimensional hyperbolic numbers have the peculiarity that the set of numbers of the first row of the matrix is completely repeated in each subsequent row with some permutation or "shift". This permutation is called the dyadic shift and is associated with the well-known operation of modulo-2 addition described below. Matrices constructed by this principle are called dyadic shift matrices. Matrix representations of $2^{n}$-dimensional hyperbolic numbers are constructed by analogy with dyadic shift matrices. Decompositions of such matrices provide that each of appearing sparse matrices contains only one identical non-zero number in each row (Figs. 2.3, 2.4, 7.2, 8.3).

Modulo-2 addition is utilized broadly in the theory of discrete signal processing as a fundamental operation for binary variables. By definition, the modulo-2 addition of two numbers written in binary notation is made in a bitwise manner in accordance with the following rules:

$$
0+0=0,0+1=1,1+0=1,1+1=0
$$


For example, modulo-2 addition of two binary numbers 110 and 101, which are equal to 6 and 5 respectively in decimal notation, gives the result $110 \oplus 101=011$, which is equal to 3 in decimal notation ( $\oplus$ is the symbol for modulo- 2 addition). The set of binary numbers

$$
000,001,010,011,100,101,110,111
$$

forms a diadic group with 8 members, in which modulo-2 addition serves as the group operation [Harmuth, 1989]. By analogy dyadic groups of binary numbers with $2^{n}$ members can be presented. The distance in this symmetry group is known as the Hamming distance. Since the Hamming distance satisfies the conditions of a metric group, the dyadic group is a metric group. The modulo-2 addition of any two binary numbers from (A2) always gives a new number from the same series. The number 000 serves as the unit element of this group: for example, $010 \oplus 000=010$. The reverse element for any number in this group is the number itself: for example, $010 \oplus 010=000$. Each member from (A2) possesses its inverse-symmetrical partner (or a mating number), which arises if the binary symbol of the member is transformed by the inverse replacements $0 \rightarrow 1$ and $1 \rightarrow 0$. For example, binary numbers 010 and 101 give an example of such pair of mating numbers.

The series (A2) is transformed by modulo-2 addition with the binary number 001 into a new series (A3) of the same numbers:

$$
\text { 001, 000, 011, 010, 101, 100,111, } 110
$$

Such changes in the initial binary sequence, produced by modulo- 2 addition of its members with any binary numbers (A2), are termed dyadic shifts [Ahmed and Rao, 1975; Harmuth, 1989]. If any system of elements demonstrates its connection with dyadic shifts, it indicates that the structural organization of its system is related to the logic of modulo-2 addition. The article shows additionally that the structural organization of genetic systems is related to logic of modulo-2 addition.

By means of dyadic groups, a special family of $\left(2^{n * 2^{n}}\right)$-matrices can be constructed which are termed "matrices of dyadic shifts" and which are used widely in technology of discrete signal processing [Ahmed, Rao, 1975; Harmuth, 1977, §1.2.6]. Fig. A1 shows examples of bisymmetric matrices of dyadic shifts. In these matrices their rows and columns are numerated by means of binary numbers of an appropriate dyadic group. All matrix cells are numerated by means of binary numbers of the same dyadic group in such way that a binary numeration of each cell is a result of modulo- 2 addition of binary numerations of its column and its row. For example, the cell from the column 110 and the row 101 obtains the binary numeration 011 by means of such addition. Such numerations of matrix cells are termed "dyadic-shift numerations" (or simply "dyadic numeration”).

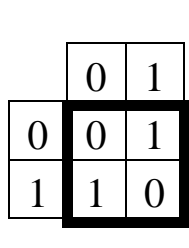

\begin{tabular}{|l|l|l|l|l|}
\cline { 2 - 5 } \multicolumn{1}{c|}{} & $00(0)$ & $01(1)$ & $10(2)$ & $11(3)$ \\
\hline $00(0)$ & $00(0)$ & $01(1)$ & $10(2)$ & $11(3)$ \\
\hline $01(1)$ & $01(1)$ & $00(0)$ & $11(3)$ & $10(2)$ \\
\hline $10(2)$ & $10(2)$ & $11(3)$ & $00(0)$ & $01(1)$ \\
\hline $11(3)$ & $11(3)$ & $10(2)$ & $01(1)$ & $00(0)$ \\
\hline
\end{tabular}




\begin{tabular}{|l|l|l|l|l|l|l|l|l|}
\cline { 2 - 9 } \multicolumn{1}{c|}{} & $000(0)$ & $001(1)$ & $010(2)$ & $011(3)$ & $100(4)$ & $101(5)$ & $110(6)$ & $111(7)$ \\
\hline $000(0)$ & $000(0)$ & $001(1)$ & $010(2)$ & $011(3)$ & $100(4)$ & $101(5)$ & $110(6)$ & $111(7)$ \\
\hline $001(1)$ & $001(1)$ & $000(0)$ & $011(3)$ & $010(2)$ & $101(5)$ & $100(4)$ & $111(7)$ & $110(6)$ \\
\hline $010(2)$ & $010(2)$ & $011(3)$ & $000(0)$ & $001(1)$ & $110(6)$ & $111(7)$ & $100(4)$ & $101(5)$ \\
\hline $011(3)$ & $011(3)$ & $010(2)$ & $001(1)$ & $000(0)$ & $111(7)$ & $110(6)$ & $101(5)$ & $100(4)$ \\
\hline $100(4)$ & $100(4)$ & $101(5)$ & $110(6)$ & $111(7)$ & $000(0)$ & $001(1)$ & $010(2)$ & $011(3)$ \\
\hline $101(5)$ & $101(5)$ & $100(4)$ & $111(7)$ & $110(6)$ & $001(1)$ & $000(0)$ & $011(3)$ & $010(2)$ \\
\hline $110(6)$ & $110(6)$ & $111(7)$ & $100(4)$ & $101(5)$ & $010(2)$ & $011(3)$ & $000(0)$ & $001(1)$ \\
\hline $111(7)$ & $111(7)$ & $110(6)$ & $101(5)$ & $100(4)$ & $011(3)$ & $010(2)$ & $001(1)$ & $000(0)$ \\
\hline
\end{tabular}

Fig. A1. The examples of matrices of dyadic shifts. Parentheses contain expressions of the numbers in decimal notation.

\section{Acknowledgments}

Some results of this paper have been possible due to a long-term cooperation between Russian and Hungarian Academies of Sciences on the theme "Non-linear models and symmetrologic analysis in biomechanics, bioinformatics, and the theory of selforganizing systems", where the author was a scientific chief from the Russian Academy of Sciences. The author is grateful to G. Darvas, E. Fimmel, A.A. Koblyakov, M. He, Z.B. Hu, Yu.I. Manin, D.G. Pavlov, I.S. Soshinsky, I.V. Stepanyan, V.I. Svirin and G.K. Tolokonnikov for their collaboration.

\section{References}

Ahmed, N.U., Rao, K.R. Orthogonal transforms for digital signal processing. SpringerVerlag New York, Inc. (1975).

Apel W. Harvard Dictionary of Music. Cambridge, Massachusetts: Harvard University Press (1969). ISBN 978-0-674-37501-7.

Arnold V. A complexity of the finite sequences of zeros and units and geometry of the finite functional spaces. Lecture at the session of the Moscow Mathematical Society, (May 13, 2007), http://elementy.ru/lib/430178/430281.

Baines A. Woodwind Instruments and Their History. New York: Courier Dover Publications (1991), ISBN 978-0-486-26885-9].

Balonin N.A. New course on the theory of motion control (Novyi kurs teorii upravleniia dvizheniem). Saint Petersburg State University, Saint Petersburg (2000) (in Russian).

Bellman R. Introduction to Matrix Analysis. N-Y: Mcgraw-Hill Book Comp. (1960)]

Bodnar O.Ya. Geometry of phyllotaxis. Reports of the Academy of Sciences of Ukraine, №9, pp. 9-15 (1992).

Bodnar O.Ya. Golden Ratio and Non-Euclidean Geometry in Nature and Art. Lviv: Publishing House "Sweet" (1994). 
34

Chargaff E. Preface to a Grammar of Biology: A hundred years of nucleic acid research. Science, 172, 637-642 (1971).

Christidis A.-F., Arapopoulou M., Christi M. A History of Ancient Greek: From the Beginnings to Late Antiquity. Cambridge: Cambridge University Press (2007). ISBN 978-0-521-83307-3.

Gladwell G.M.L. Inverse Problems in Vibration. London, Kluwer Academic Publishers, (2004).

Collins Concise Dictionary, 4th edition, HarperCollins, Glasgow (1999). ISBN 000 4722574.

Day-O'Connell J. Pentatonicism from the Eighteenth Century to Debussy. Rochester: University Rochester Press (2007). ISBN 978-1-58046-248-8.

Fimmel E., Danielli A., Strüngmann L. On dichotomic classes and bijections of the genetic code. J. Theor. Biol., 336, 221-230. https://doi.org/10.1016/j.jtbi.2013.07.027 (2013)

Frey-Wissling A., Muhlethaler K. Ultrastructural plant cytology, with an Introduction to molecular biology. Amsterdam, New York, Elsevier Pub. Co. (1965).

Gsponer A., Hurni J.-P. Quaternions in mathematical physics (2): Analytical bibliography, http://arxiv.org/abs/math-ph/0511092, 3th version from 6 July 2008.

Harkin A.A., Harkin J.B. Geometry of Generalized Complex Numbers. Mathematics Magazine, v. 77(2), p. 118-29 (2004).

Harmuth, H. F. Sequency theory. Foundations and applications. N.-Y.: Academic Press. (1977).

Harmuth, H. F. Information theory applied to space-time physics. Washington: The Catholic University of America, DC (1989)

Heisenberg W. Physics and philosophy. New York: Harper \& Row, 1958.

Hesse H. The Glass Bead Game. St Martins Press (2002), ISBN 0312278497, 9780312278496]).

Jean R. Phyllotaxis. A systemic study in plant morphogenesis. Cambridge Univ. Press (2006).

Kantor I.L., Solodovnikov A.S. Hypercomplex numbers. Berlin, New York: SpringerVerlag (1989). ISBN 978-0-387-96980-0.

Klein F. Elementary Mathematics from an Advanced Standpoint: Arithmetic, Algebra, Analysis. London: Dover Publications (2004).

Kline M. Mathematics: The Loss of Certainty. Oxford University Press (1982).

May R.M. Uses and Abuses of Mathematics in Biology. Science, v. 303, pp. 790-793 (6 February 2004).

Nielsen M.A., Chuang I.L. Quantum Computation and Quantum Information. New York: Cambridge University Press. https://doi.org/10.1017/CBO9780511976667 (2010).

Olsen D.A., Sheehy D.E. (Eds.) The Garland Encyclopedia of World Music, Volume 2: South America, Mexico, Central America, and the Caribbean. New York: Taylor \& Francis. (1998). ISBN 0824060407.

Pauling L. The Nature of the Chemical Bond and the Structure of Molecules and Crystals: An Introduction to Modern Structural Chemistry, 2nd ed. Oxford University Press, 
London, 664 p. (1940).

Pavlov D.G., Panchelyuga M.S., Panchelyuga V.A. On the form of the analogue of the Julia set at zero value of the parameter on the plane of the double variable. Hypercomplex numbers in geometry and physics, v. 6, N 1 (11), pp. 146-151 (2009a).

Pavlov D.G., Panchelyuga M.S., Panchelyuga V.A. On the form of analogues of the Julia set on the plane of a double variable. Hypercomplex numbers in geometry and physics, v. 6, N 2 (12), pp. 161-175 (2009b).

Petoukhov S.V. Biomechanics, bionics and symmetry. Moscow, Nauka (1981)

Petoukhov S.V. Biosolitons. Moscow: GP Kimrskaya tipographia, 288p. (1999).

Petoukhov S.V. Matrix genetics, algebrases of genetic code, noise immunity. Moscow, RCD, 316 p. (2008) (in Russian).

Petoukhov S.V. Matrix genetics and algebraic properties of the multi-level system of genetic alphabets. Neuroquantology, v. 9, №4, p. 60-81 (2011).

Petoukhov S.V. The concept of resonances in genetics ("resonant bioinformatics"). Selected proceedings of the VII International Congress "Weak and Super-Weak Fields and Radia tions in Biology and Medicine", 7. 09.2015, St. Petersburg, pp.1-41 (2015). http://www.biophys.ru/lib/sci/resonance/460-resonance-00001.

Petoukhov S.V. The system-resonance approach in modeling genetic structures. Biosystems, v. 139, p. 1-11 (January 2016).

Petoukhov S.V. The Genetic Coding System and Unitary Matrices. Preprints 2018, 2018040131 (doi: 10.20944/preprints201804.0131.v2), submitted 27.09.2018. http://www.preprints.org/manuscript/201804.0131/v2 (2018a).

Petoukhov S.V. Structural Connections between Long Genetic and Literary Texts. Preprints 2018, 2018120142, online 1502 2019. (DOI:

10.20944/preprints201812.0142.v2) (2018b). https://www.preprints.org/manuscript/201812.0142/v2

Petoukhov S.V. Nucleotide Epi-Chains and New Nucleotide Probability Rules in Long DNA Sequences. Preprints 2019, 2019040011 (doi: 10.20944/preprints201904.0011.v2) (2019a). https://www.preprints.org/manuscript/201904.0011/v2.

Petoukhov S.V. Connections Between Long Genetic and Literary Texts. The QuantumAlgorithmic Modelling. In: Hu Z., Petoukhov S., Dychka I., He M. (eds) Advances in Computer Science for Engineering and Education II. pp 534-543, ICCSEEA 2019. Advances in Intelligent Systems and Computing, vol. 938. Springer, Cham. Online publication on 29 March 2019. (2019b). https://link.springer.com/chapter/10.1007/978-3-030-16621-2_50\#citeas .

Petoukhov S.V., He M. Symmetrical Analysis Techniques for Genetic Systems and Bioinformatics: Advanced Patterns and Applications. IGI Global, USA (2010). http://petoukhov.com/Petoukhov, \%20He\%20-\%202010\%20$\%$ 20Symmetrical\%20Analysis $\% 20$ Techniques $\% 20$ for $\% 20$ Genetic $\% 20$ Systems $\% 20$ and $\% 20 \mathrm{~B}$ ioinformatics.pdf.

Petoukhov S.V., Petukhova E.S., Svirin V.I. Symmetries of DNA alphabets and quantum informational formalisms. Symmetry: Culture and Science, Vol. 30, No. 2, 161-179 
36

(2019), https://doi.org/10.26830/symmetry_2019_2_161

Rashevskij P.K. Tensor analysis and Riemannian geometry. Moscow, Nauka (1964).

Russell B. A history of western philosophy. New York: Touchstone (1945).

Sethares W.A. Tuning, Timbre, Spectrum, Scale. London, Springer, p.163 (2005). ISBN 1-85233-797-4.

Shervatov V.G. Hyperbolic functions. Moscow, GITTL (1954).

Shults E. The organism as a creativity. In the book "Questions of theory and psychology of creativity" ("Voprosy teorii i psikhologii tvorchestva"), Russia, Kharkov, vol.7, pp. 108190 (1916) (in Russian)].

Smolyaninov V.V. Spatio-temporal problems of locomotion control. Uspekhi Fizicheskikh Nauk, v. 170, N 10, pp. 1063-1128 (2000), DOI: https://doi.org/10.3367/UFNr.0170.200010b.1063.

Stakhov A.P. The Mathematics of Harmony. From Euclid to Contemporary Mathematics and Computer Science. New Jersey, World Scientific (2009).

Stambuk N. Circular coding properties of gene and protein sequences. Croat. Chem. Acta, 72, pp. 999-1008 (1999).

Sudhibhushan Bhattacharya. Ethno-musicology and India. Indian Publications, p. 54 (1968). Retrieved 22 June 2012.

Todd Titon J.T. Worlds of Music: An Introduction to the Music of the World's Peoples, Shorter Version. Boston: Cengage Learning (1996). ISBN 9780028726120.

Vernadsky V.I. The chemical structure of the biosphere and its environment. Moscow, Nauka (1965) (in Russian).

Weinberger N.M. Music and brain. Sci. Amer., 291 (5), pp. 88-95 (2004).

Yamagishi M.E.B. Mathematical Grammar of Biology. - Switzerland, Springer International Publishing AG (2017). ISBN 978-3-319-62689-5.

Zharov V.E. Spherical astronomy. Friazino: RFFI (2002). 